ARTICLE

https://doi.org/10.1038/s41467-019-11246-1

\title{
RIF1 promotes replication fork protection and efficient restart to maintain genome stability
}

\author{
Chirantani Mukherjee ${ }^{1}$, Vivek Tripathi ${ }^{1}$, Eleni Maria Manolika' ${ }^{1}$ Anne Margriet Heijink ${ }^{2,5}$, Giulia Ricci ${ }^{1}$,

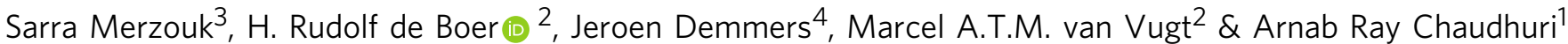

Homologous recombination (HR) and Fanconi Anemia (FA) pathway proteins in addition to their DNA repair functions, limit nuclease-mediated processing of stalled replication forks. However, the mechanism by which replication fork degradation results in genome instability is poorly understood. Here, we identify RIF1, a non-homologous end joining (NHEJ) factor, to be enriched at stalled replication forks. Rif 1 knockout cells are proficient for recombination, but displayed degradation of reversed forks, which depends on DNA2 nuclease activity. Notably, RIF1-mediated protection of replication forks is independent of its function in NHEJ, but depends on its interaction with Protein Phosphatase 1. RIF1 deficiency delays fork restart and results in exposure of under-replicated DNA, which is the precursor of subsequent genomic instability. Our data implicate RIF1 to be an essential factor for replication fork protection, and uncover the mechanisms by which unprotected DNA replication forks can lead to genome instability in recombination-proficient conditions.

\footnotetext{
${ }^{1}$ Department of Molecular Genetics, Erasmus University Medical Center, Wytemaweg 80, Rotterdam 3015CN, The Netherlands. ${ }^{2}$ Department of Medical Oncology, University Medical Center Groningen, University of Groningen, Hanzeplein 1, 9713 GZ Groningen, The Netherlands. ${ }^{3}$ Department of

Developmental Biology, Erasmus University Medical Center, Wytemaweg 80, Rotterdam 3015CN, The Netherlands. ${ }^{4}$ Department of Biochemistry, Erasmus University Medical Center, Wytemaweg 80, Rotterdam 3015CN, The Netherlands. ${ }^{5}$ Present address: Lunenfeld-Tanenbaum Research Institute, Mount Sinai Hospital, Toronto, ON M5G 1X5, Canada. Correspondence and requests for materials should be addressed to A.R.C. (email: a.raychaudhuri@erasmusmc.nl)
} 
P roteins involved in the $\mathrm{HR}$ and FA pathways like BRCA1/2 and FANCD2 have been associated with repair of replication-associated DNA damage ${ }^{1,2}$. Additionally, HR and FA factors protect DNA replication forks from extensive MRE11 nuclease-mediated degradation, preventing genome instability ${ }^{3,4}$. This function is clinically relevant as fork protection was found to induce chemoresistance in BRCA2-defective cells ${ }^{5,6}$. Another parallel pathway in the processing of stalled replication forks has been identified, involving the DNA2 nuclease ${ }^{7,8}$.

Recently, replication fork reversal was shown to be required for effective fork degradation in BRCA2-deficient cells, with the "regressed arm" being the access point for MRE11-mediated processing ${ }^{9-12}$. Although fork reversal is a stabilizing structure for stalled replication forks ${ }^{13-16}$, degradation of regressed forks results in genome instability ${ }^{9-12}$. However, the mechanisms that regulate fork degradation-mediated genome instability remain poorly understood.

Mammalian Rap1-interacting factor 1 (RIF1) has multiple functions, including mediating NHEJ at double strand breaks (DSBs), regulation of replication origin timing, and resolution of catenanes ${ }^{17-25}$. In the process of DSB repair via NHEJ, RIF1 is a crucial interactor of $53 \mathrm{BP} 1^{17,19-21,25,26}$ and interacts with the $\mathrm{N}$-terminal SQ/TQ sites of $53 \mathrm{BP} 1^{26}$. Loss of RIF1 also results in resistance to PARP inhibitor treatment signifying its clinical relevance ${ }^{17,20,21}$

RIF1 has also been implicated in the control of replication timing in mammalian cells ${ }^{18}$, mediated through its interaction with Protein Phosphatase 1 (PP1) 22,24 . Interestingly, Rif1-deficient mice are embryonic lethal, suggesting that RIF1 could be involved in the tolerance of high levels of replicative stress encountered during proliferation of stem cells ${ }^{27,28}$.

Here, we show a novel role for RIF1 in the protection of reversed replication forks from DNA2-mediated degradation. Furthermore, the C-terminal domain of RIF1-responsible for binding both protein phosphatase 1 as well as cruciform DNA structures-is essential for protecting reversed forks from degradation. Finally, we provide evidence that degradation of reversed forks is linked to defective replication restart in RIF1deficient cells, resulting in the accumulation of under-replicated DNA and subsequent genome instability.

\section{Results}

RIF1 is recruited to stalled DNA replication forks. To identify novel factors enriched at stalled replication forks, we utilized iPOND (isolation of proteins on nascent DNA) coupled with SILAC (stable isotope labeling of amino acids in cell culture)-based quantitative mass-spectrometry ${ }^{29,30}$. Mouse embryonic stem cells were treated with hydroxyurea (HU) to stall DNA replication forks and subsequently subjected to quantitative mass-spectrometry to analyze the proteomes associated with the replication forks (Fig. 1a and Supplementary Data 1). Seven-hundred twenty-one proteins were identified commonly between two independent experiments (Supplementary Fig. 1a). We identified RIF1 among 44 proteins, which showed $>2$-fold enrichment upon HU treatment (Fig. 1b and Supplementary Data 1). Consistent with previous reports, we also observed over two-fold increase in replication stress response proteins, including RAD51 and RPA2 (Fig. 1c and Supplementary Data 1$)^{29,30}$. Whereas core components of the replicative helicase, including MCM2-7, largely remained unchanged at time of early replication stress, PCNA enrichment decreased at stalled replication forks, as reported previously ${ }^{30}$ (Fig. 1c).

To further verify the recruitment of RIF1 to stalled forks, we performed immunofluorescence analysis to measure localization of RIF1 at sites of DNA replication. Wild type (WT) mouse embryonic fibroblasts (MEFs) were incubated with EdU, and localization of RIF1 to sites of EdU incorporation was measured in the presence or absence of $\mathrm{HU}$ treatment (Fig. 1d). Approximately $50 \%$ of the WT cells in non-treated condition (NT) showed EdU incorporation, (Fig. 1d, e). Only a small fraction of EdU-positive WT cells in non-treated cells were positive for RIF1 foci. By contrast, upon HU treatment, approximately $80 \%$ of the EdU-positive cells showed EdU colocalization with RIF1 (Fig. 1d, e and Supplementary Fig. 1c). To verify that EdU and RIF1 co-localization upon HU treatment indeed occurred at stalled forks, we performed proximity ligationbased assays (PLA) to detect RIF1 binding to replicated DNA ${ }^{12}$. WT cells treated with HU displayed a significant increase in PLA signals per cell. However, the total percentage of PLA-positive cells (signifying the replicating population) did not increase significantly (Supplementary Fig. 1d), suggesting that RIF1 is recruited to stalled DNA replication forks.

Since RIF1 localization to sites of DSBs depends on 53BP1 17,19-21,25,26 (Supplementary Fig. 1b, e), we tested if localization of RIF1 to stalled replication forks also required 53BP1. Interestingly, upon $\mathrm{HU}$ treatment $53 b p 1^{-1-} \mathrm{MEFs}$ showed similar levels of RIF1-EdU co-localization as WT cells (Fig. 1d, e and Supplementary Fig. 1b, c). Finally, we tested whether 53BP1 also localized to sites of DNA stalled forks upon HU treatments. In WT MEFs we observed 53BP1 foci in a low percentage of cells, but these foci did not co-localize with EdU (Supplementary Fig. 1f). Furthermore, HU treatments did not significantly increase the percentage of 53BP1-positive cells (Supplementary Fig. 1f), suggesting that RIF1 is enriched at stalled replication forks, independently of 53BP1.

RIF1 protects reversed DNA replication forks. To explore the role of RIF1 during unperturbed DNA replication, we monitored the frequency of replicating cells by incorporation of EdU by flow cytometry (Supplementary Fig. 2a-d). WT and Rif1 $1^{-1-}$ cells showed similar percentages and intensities of EdU staining (Supplementary Fig. 2b-d). Additionally, we analyzed progression rates of individual replication forks in WT and Rif1 ${ }^{-1-}$ cells by DNA fiber assay. We sequentially labeled cells with CldU (red) and IdU (green), followed by tract length analysis (Fig. 2a and Supplementary Fig. 7a). WT and Rif1 ${ }^{-1-}$ cells revealed no significant difference in tract lengths, again suggesting that RIF1 is not essential for unperturbed DNA replication (Fig. 2a).

Next, we tested if RIF1 was involved in stabilizing DNA replication forks under stressed conditions. WT and Rif1-/MEFs were sequentially labeled with CldU and IdU. On-going replication forks were then stalled with $\mathrm{HU}$ (Fig. 2b). The relative shortening of the IdU tract after HU treatment served as a measure of fork degradation (Fig. 2b). Upon HU treatment, WT cells showed tract lengths similar to non-treated cells with mean ratio close to 1 (Fig. 2b). Contrastingly, RIF1-deficient cells displayed a significant reduction in the IdU tract lengths (Fig. $2 \mathrm{~b}$ and Supplementary Fig. 7b). Human RIF1 knock-out HAP1 cells $(\mathrm{RIF} 1-\mathrm{KO})^{23}$, also revealed a similar trend as observed in Rif1 $1^{-/-}$ MEFs (Fig. 2c and Supplementary Figs. 2e and 7c). This suggests that RIF1 is essential for protection of replication forks from degradation (Fig. 2b, c). Recently, 53BP1 deficiency in B-lymphocytes was demonstrated to cause degradation of nascent strands ${ }^{31}$. Since RIF1 interacts with 53BP1 for NHEJ, we tested whether protection of nascent strands by RIF1 could be dependent on this interaction. Analysis of 2 different clones of $53 b p 1^{-/-}$MEFs did not show fork degradation upon HU treatment. This suggests that in our experimental setup, the role of RIF1 in replication fork protection is independent of 53BP1 (Fig. 2d and Supplementary Fig. 7d). 
a

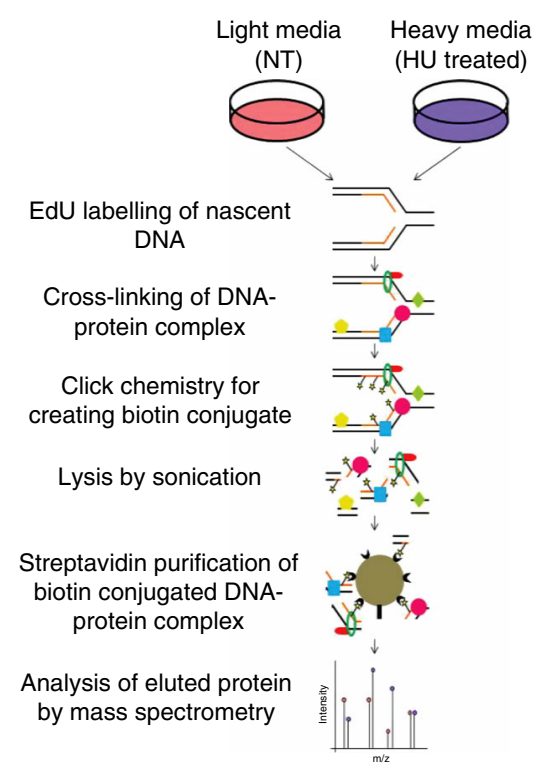

C

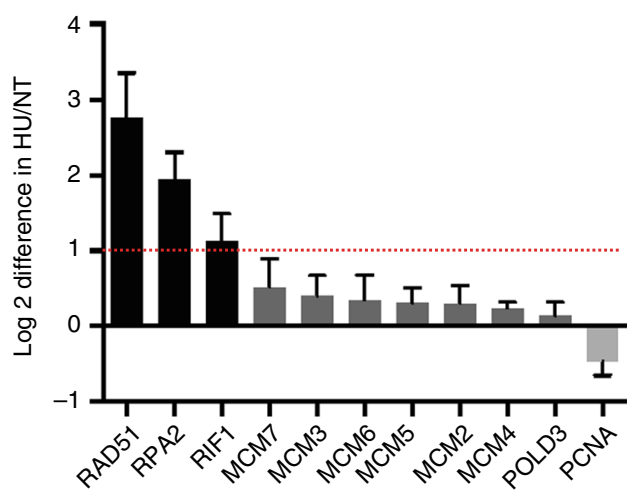

$\mathbf{e}$

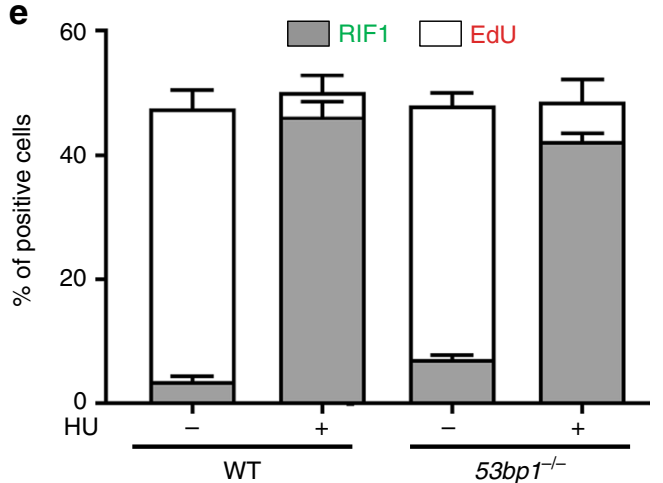

b

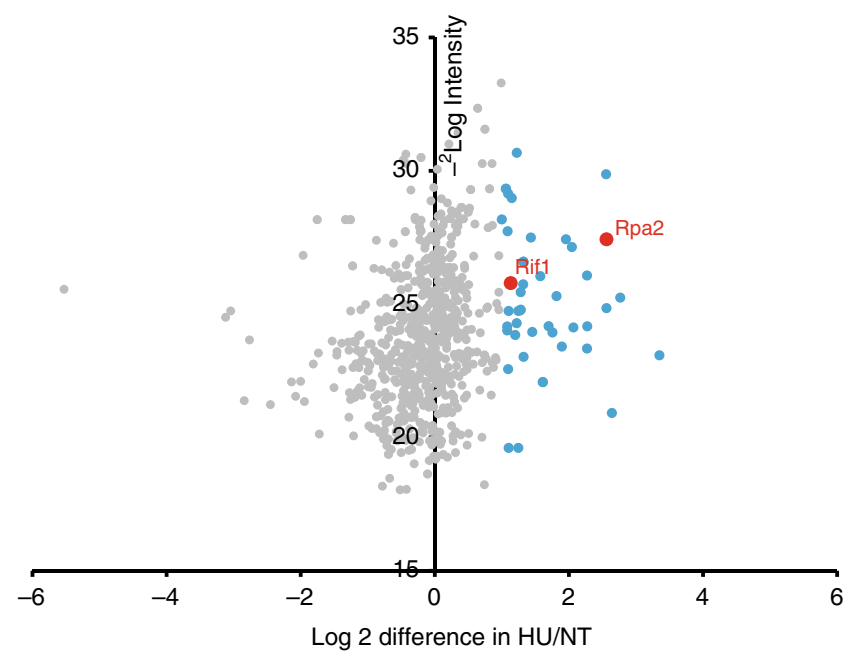

d

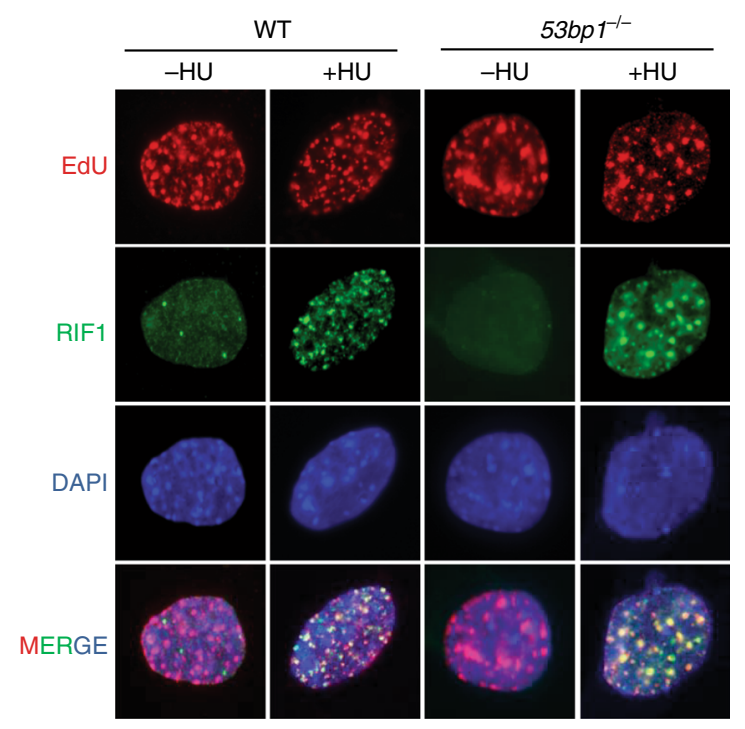

Fig. 1 RIF1 is recruited to the stalled replication forks. a Schematic representation of iPOND experiment. b Volcano plot showing the results for average fold-change to identify significantly upregulated proteins upon $\mathrm{HU}$ treatment based on H:L ratio in the SILAC experiment. The $x$-axis ('2Log Difference HU/NT) represents the fold upregulation. Data points in blue represent proteins that are upregulated $>2$-fold; RIF1 is indicated in red. c Bar graph showing fold upregulation of a selection of proteins upon HU treatment based on their SILAC H:L ratios (error bars represent standard deviation). d Representative micrographs showing co-localization of RIF1 (green) to sites of DNA replication as marked by EdU (red) in the presence or absence of $\mathrm{HU}$ in WT and $53 \mathrm{bp} 1^{-1-}$ cells. Nucleus was stained with DAPI (blue). e Quantitation of $\mathbf{d}$ showing the percentage of cells, which show colocalization between EdU and RIF1(error bars represent standard deviation) 
a
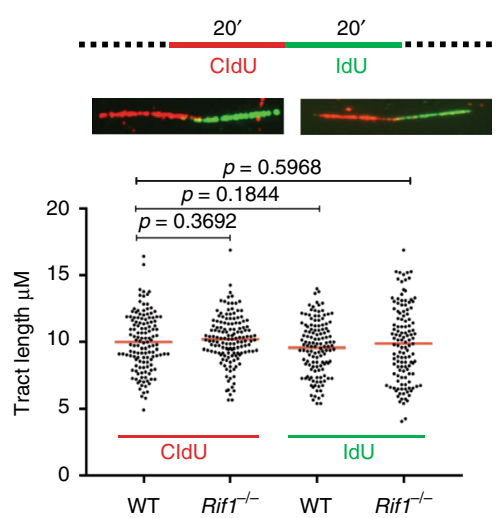

C

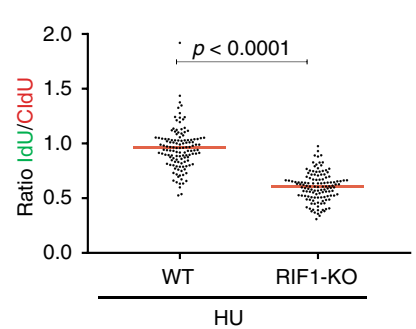

e
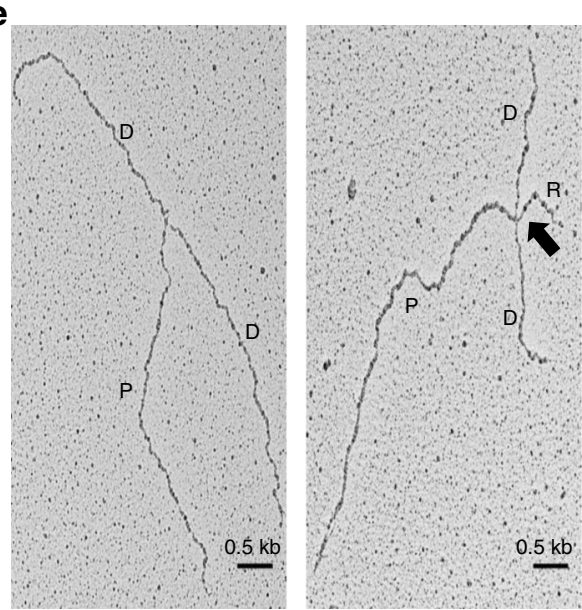

g

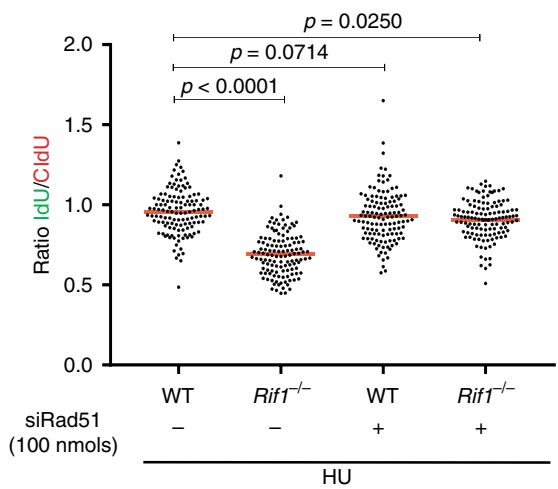

b
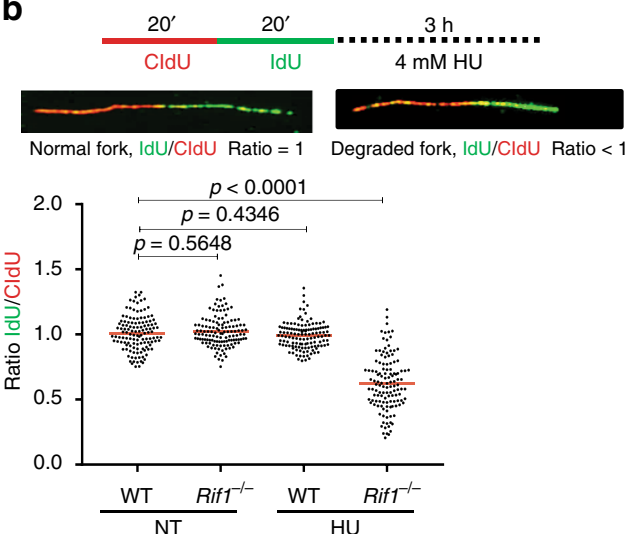

d

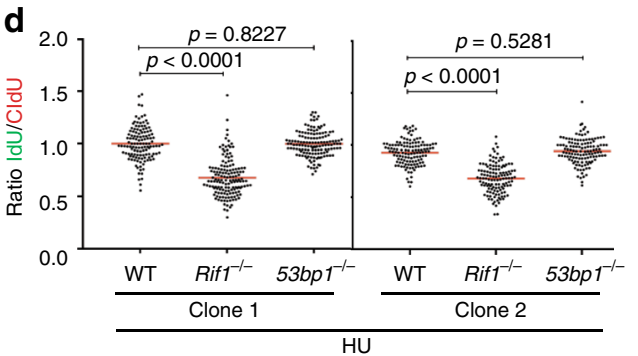

$\mathbf{f}$

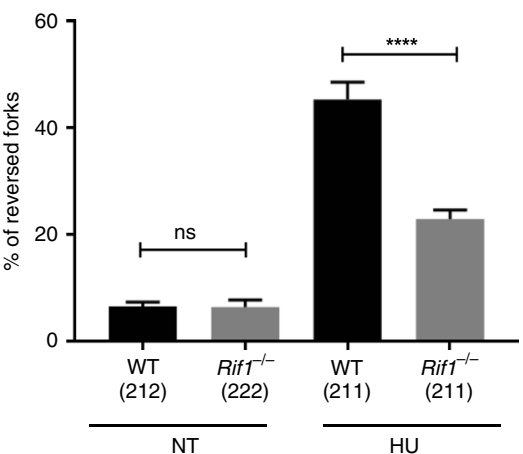

h

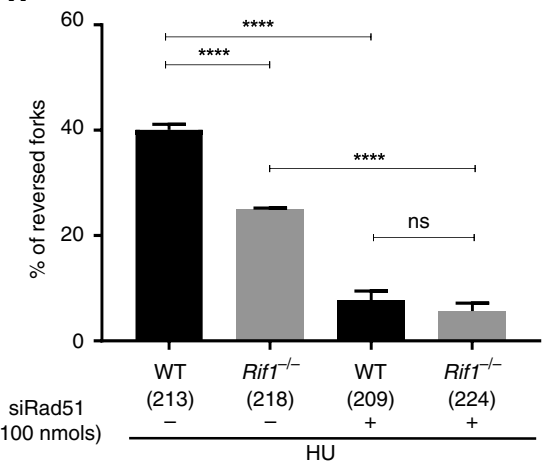

Fork degradation has been associated with loss of HR factors ${ }^{3,4}$. We, therefore, tested if loss of RIF1 also resulted in HR defects. Localization of the RAD51 recombinase to sites of DNA DSBs has been shown to be a reliable readout for functional $\mathrm{HR}^{32}$. Upon ionizing irradiation, Rif $1^{-1-}$ MEFs were proficient in forming RAD51 foci (Supplementary Fig. 3a, b). Additionally, we monitored HR efficiency using the DR-GFP reporter ${ }^{33}$. Consistent with earlier reports ${ }^{20,34,35}$, Rif1 $^{-/}$MEFs did not show a significant difference in HR frequencies when compared to WT cells (Supplementary Fig. 3c). Finally, we tested the ability of RIF1-deficient cells to form sister chromatid exchanges (SCEs) in the presence or absence of HU or cisplatin. Treatments with 
Fig. 2 Protection of reversed forks from degradation by RIF1. a Top panel: schematics of experimental conditions for fork progression in WT and Rif1-/MEFs. Cells were labeled with CldU (red) followed by IdU (green) as indicated. Representative DNA fibers for progression in WT and Rif1 ${ }^{-1-}$ MEFs are shown below the schematic. Progression was measured by tract lengths of CldU (red) and IdU (green) in micrometers ( $\mu$ M). b Top panel: schematic for labeling cells in fork degradation assay. Representative pictures of normal and degraded fork are shown below the schematic. Cells were labeled with CldU followed by IdU and then subjected to replication stress with $4 \mathrm{mM} \mathrm{HU}$ for $3 \mathrm{~h}$. Ratio of IdU to CldU tract length was plotted as readout for fork degradation. c, d Fork degradation assay in WT and RIF1-KO HAP1 cells (c) and between two different clones of WT, Rif1-/-, and 53bp $1^{-/-}$MEF cell line (d). Experimental conditions were similar as in $\mathbf{b}$. e Representative electron micrographs of normal fork (left) and reversed replication fork (right) observed on treatment with HU. The black arrow pointing to four-way junction at the replication fork indicates fork reversal (P, Parental, D, Daughter strand, R, Reversed arm). f Percentage of fork reversal in WT and Rif1-/- MEFs treated with or without HU (4 mM) for $3 \mathrm{~h}$. Numbers of analyzed molecules are indicated in parentheses. $\mathbf{g}$ WT and Rif1-/- MEFs were transfected with siRad51 (100 nmols, $48 \mathrm{~h}$ ) followed by labeling and treatment with 4 mM HU for $3 \mathrm{~h}$. Fork degradation was determined in the presence and absence of RAD51. $\mathbf{h}$ Fork reversal frequencies observed with and without depletion of RAD51 in WT and Rif1 ${ }^{-1}$ MEFs under HU treatment. Numbers of analyzed molecules are indicated within parenthesis. Red bars in $\mathbf{a}, \mathbf{b}, \mathbf{c}, \mathbf{d}$, and $\mathbf{g}$ represent mean values from 125 fibers from each genotype under each condition. P-values were derived from Kruskal-Wallis ANOVA with Benjamini Hochberg (BH) post test except in $\mathbf{c}$, where Mann-Whitney was used and in $\mathbf{f}$ and $\mathbf{h}$, where unpaired $t$-test was done (ns, non-significant, ${ }^{\star \star \star \star} P<0.0001$ ). All experiments were repeated three times with similar outcomes (Supplementary Data 2 and Supplementary Fig. 7a-e)

either HU or cisplatin significantly increased the number of SCEs in both WT and Rif1-l- cells. However, no significant differences in SCEs were observed between WT and Rifi $^{-1-}$ cells (Supplementary Fig. 3d-e). Taken together, these data suggest that loss of RIF1 does not result in defective HR.

DNA replication stress results in fork reversal ${ }^{14}$. Recent reports have identified reversed forks to be the substrate for nascent stand degradation in the absence of $\mathrm{BRCA} 2^{9-12}$. We, therefore, hypothesized that RIF1 -like BRCA2- could be involved in the protection of reversed forks. To assess replication fork architecture in WT and Rifl $^{-1-}$ cells, we visualized replication intermediates formed in vivo using electron microscopy $(\mathrm{EM})^{36}$. HU treatment of WT MEFs resulted in a high percentage of reversed replication forks (Fig. 2e, f and Supplementary Data 3). In contrast, HUtreated RIF1-deficient cells showed a significantly lower frequency of fork reversal (Fig. $2 \mathrm{f}$ and Supplementary Data 3). These data suggest that RIF1 could either be involved in mediating fork reversal or in protecting reversed forks.

RAD51 has been shown to be essential for mediating fork reversal 11,37,38. RAD51 downregulation rescues fork degradation in BRCA2-deficient cells, suggesting that unprotected reversed forks are the substrates for degradation ${ }^{10,11,39}$. However, stabilization of RAD51 on the reversed forks is also important for protection of reversed forks ${ }^{40}$. To test if RIF1 is involved in fork reversal, we downregulated RAD51 in WT and RIF1deficient MEFs and tested for fork degradation (Fig. 2g and Supplementary Fig. 3f). Near-complete downregulation of RAD51 in WT cells did not induce fork degradation in WT cells (Fig. 2g) ${ }^{10,11,39}$. However, RAD51 downregulation in Rif1 $1^{-1-}$ cells significantly rescued fork degradation, suggesting that RIF1 is required for fork protection but not for reversal of forks (Fig. 2g and Supplementary Fig. 3f, 7e). Consistently, our EM analysis showed that knockdown of RAD51 in WT cells resulted in almost complete abolishment of fork reversal upon HU treatments (Fig. 2h and Supplementary Data 3$)^{11,37,38}$. However, this decrease in fork reversal was not further affected by RIF1 inactivation (Fig. $2 \mathrm{~h}$ and Supplementary Data 3 ). To subsequently test if RIF1 acts epistatic to RAD51 in protecting reversed forks, we partially downregulated RAD51 in WT and Rif1 $1^{-1-}$ cells and assessed fork degradation (Supplementary Figs. 3g, h and 7f). Partial downregulation of RAD51 resulted in fork degradation in WT cells, but did not result in aggravated degradation observed upon RIF1-deficiency alone, suggesting that RIF1 could also be involved in the stabilization of RAD51 on the reversed arm (Supplementary Figs. 3h and 7f). Taken together, these data strongly suggest that RAD51 acts upstream of RIF1 in fork reversal and that RIF1 could be involved in the protection of reversed forks, rather than the process of fork reversal itself.

Fork degradation in RIF1-deficient cells mediated by DNA2. Since MRE11 has been implicated in mediating replication fork degradation ${ }^{3,4}$, we tested if MRE11 is also responsible for fork degradation upon RIF1- deficiency. We downregulated MRE11 in WT and Rif1 ${ }^{-1-}$ MEFs (Fig. 3a) and measured fork degradation. Downregulation of MRE11 in RIF1-deficient cells resulted in a partial but significant rescue of fork degradation (Fig. 3b and Supplementary Fig. 7g). Since partial rescue of fork degradation could result from residual MRE11 activity, we treated cells with the MRE11 inhibitor Mirin ${ }^{41}$. Mirin treatment failed to completely rescue the fork degradation phenotype in Rif1 ${ }^{-1-}$ MEFs, again suggesting that MRE11 is not the main nuclease involved in degradation of replication forks in Rif1 $1^{-/}$cells (Supplementary Fig. $4 \mathrm{a}$ and $7 \mathrm{~h}$ ). DNA2 nuclease has been implicated in the restart of reversed replication forks ${ }^{37}$ and the uncontrolled degradation of stalled replication forks ${ }^{7,8}$. Therefore, we tested if DNA2 was involved in the degradation of replication forks in Rif $1^{-1-}$ MEFs. Downregulation of DNA2 completely rescued the fork degradation in Rif1 ${ }^{-1-}$ MEFs (Fig. 3a, b). We next analyzed the involvement of DNA2 in fork degradation in RIF1-KO HAP1 cells, using the DNA2 inhibitor NSC-105808 (DNA2i) ${ }^{42}$. Pretreatment of RIF1-KO cells with DNA2i significantly rescued the degradation of nascent strands (Fig. 3c), and no additional rescue was observed upon combined Mirin and DNA2i treatments (Fig. 3c and Supplementary Fig. 7i). A dependency on DNA2 for fork degradation was also confirmed in Rif1 $1^{-1-}$ MEFs, using either Mirin, DNA2i or both (Supplementary Figs. 4a and 7h). To verify the context specificity for DNA2, we pretreated $\mathrm{Brca}^{-1-}$ MEFs with either Mirin, DNA2i or both and assessed the rescue of fork degradation. While Mirin treatment rescued fork degradation as expected (Supplementary Figs. 4b and 7j), DNA2i treatment only partially rescued fork degradation in $\mathrm{Brca}^{-1-}$ cells. Additionally, combined inhibition of MRE11 and DNA2 in $\mathrm{Brca1}^{-1-}$ cells did not show any additive effect (Supplementary Figs. 4b and 7j), suggesting that DNA2 is the main nuclease driving fork degradation in RIF1-deficient cells.

Next, to test if DNA2 inhibition could rescue formation of reversed forks upon RIF1-deficiency, cells were treated with HU in the presence or absence of DNA2i, and the frequencies of reversed forks were analyzed. As observed earlier, Rifi ${ }^{-1-}$ MEFs treated with $\mathrm{HU}$ displayed a significantly reduced frequencies of reversed forks. Strikingly, treatment with DNA2i and HU in Rif $1^{-1-}$ cells significantly rescued fork reversal (Fig. 3d and 

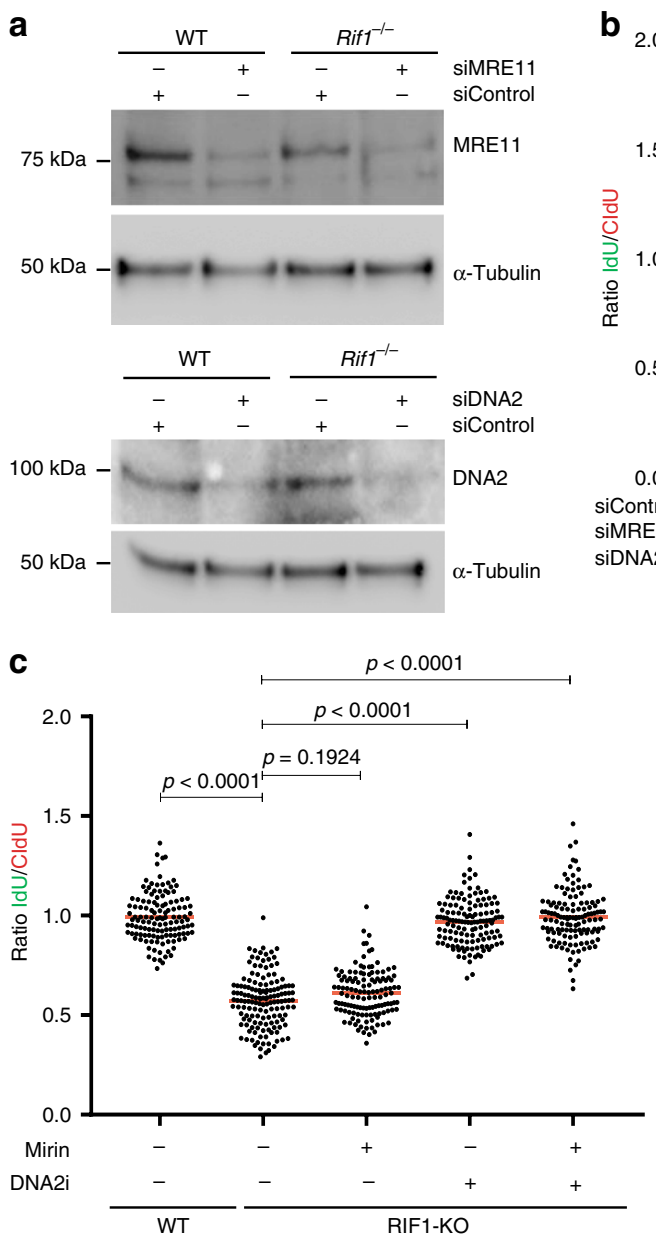

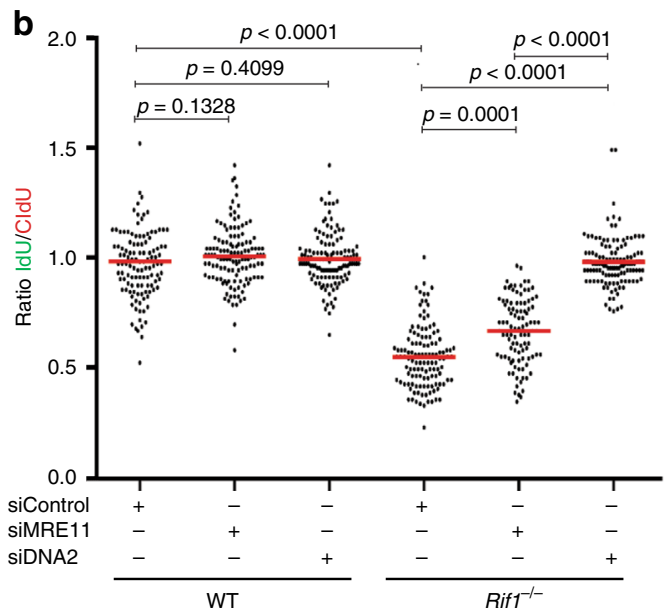

d

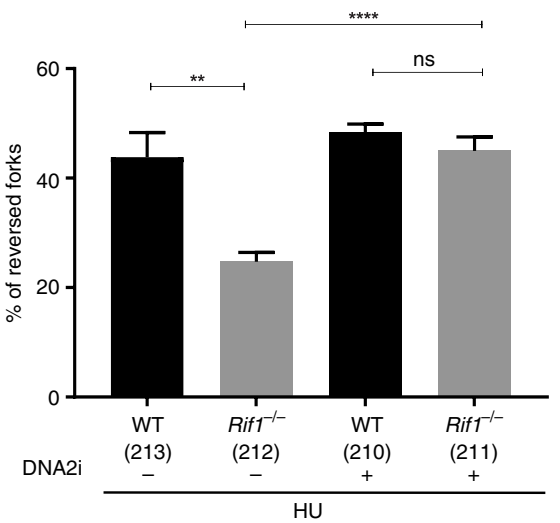

Fig. 3 DNA2 drives reversed fork degradation in RIF1-deficient cells. a Western blot analysis for the downregulation of MRE11 and DNA2 in WT and Rif1-/MEFs. WT and Rif1-/- MEfs were transfected with either siControl or siRNAs smart pool against MRE11 and DNA2. Lysates made were probed with antibody against MRE11 and DNA2. Tubulin is used as loading control. b Ratio of IdU versus CldU in WT and Rif1-/- MEFs upon HU treatment after downregulating Mre11 or DNA2 (a). c Ratio of IdU versus CIdU in WT and RIF1-KO HAP1 cells upon HU treatment after inhibiting Mre11 and DNA2 using mirin and DNA2 inhibitor. d Electron microscopic analysis of percentage of reversed forks observed in WT and Rif1-/- MEFs subjected to HU (4 mM) for $3 \mathrm{~h}$, with or without DNA2 inhibitor. Numbers of analyzed molecules are indicated in parentheses. At least 125 readings were taken for $\mathbf{b}$ and $\mathbf{c}$ and the mean ratio is represented by red bar. $P$-values were derived from Kruskal-Wallis ANOVA with Benjamini Hochberg post test except in d, where unpaired $t$-test (ns, non-significant, ${ }^{\star \star \star \star} P<0.0001,{ }^{\star \star} P=0.0024$ ) was carried out. Similar observation was made from three independent experiments

(Supplementary Data 2 and Supplementary Fig. $7 g$, i)

Supplementary Data 3). Altogether, these data show that RIF1 is responsible for protecting reversed replication forks from DNA2mediated degradation.

C-terminal region of RIF1 is essential for fork protection. Mammalian RIF1 has two conserved regions at its termini ${ }^{20}$. The $\mathrm{N}$-terminus consists of HEAT-like $\alpha$-helical repeats (HEATrepeats) and is required for Rif1 recruitment to sites of $\mathrm{DSBs}^{20}$. The C-terminal domain (CTD) of RIF1 consists of three subdomains (CI, CII and CIII) and confers in vitro DNA binding activity, preferentially to cruciform structures ${ }^{43}$. Mammalian RIF1 also contains two PP1 interaction motifs, which are responsible for the control of replication timing $24,40,43$.

To test which domain of RIF1 is responsible for protection of reversed replication forks, we generated truncation constructs from a human full-length RIF1 construct (hRIF1-FL) ${ }^{20}$. Deletion constructs were generated for the HEAT domain (Del-HEAT), CTD domain (Del-CTD), CI domain (Del-CI), and CII domain (Del-CII) (Fig. 4a). These constructs were then transfected into Rif1 $1^{-1-}$ MEFs and checked for their expression levels (Fig. 4b).
Complementation with hRIF1-FL and Del-HEAT significantly rescued the fork degradation observed in Rif1 $1^{-1-}$ MEFs (Fig. 4c). In contrast, expression of RIF1 deletion mutants with either the CI, CII domains or the whole CTD failed to rescue the fork degradation in RIF1-deficient cells (Fig. 4c and Supplementary Fig. 8a). Furthermore, complementation of Rif1-/- MEFs with either hRIF1-FL or Del-HEAT constructs resulted in a $\sim 2$-fold increased fork reversal frequency when compared with Rif1-/MEFs (Fig. 4d and Supplementary Data 3). In contrast, Rif1-/MEFs with either Del-CI or Del-CII failed to restore fork reversal frequencies in these cells (Fig. 4d). These data suggest that the CI and CII domains of RIF1, which contain interaction motifs for PP1 and have DNA cruciform binding properties, are essential for protection of reversed forks.

To directly test the involvement of PP1 in replication fork protection, we depleted PP1 in WT and Rif1-I- MEFs and assessed fork degradation (Supplementary Fig. 4c). Interestingly, depletion of PP1 in WT cells resulted in significant fork degradation upon HU treatments, which was epistatic with RIF1 (Fig. 4e and Supplementary Fig. 8b). Furthermore, pretreatment of WT and Rif1-/- MEFs with the selective PP1 
a
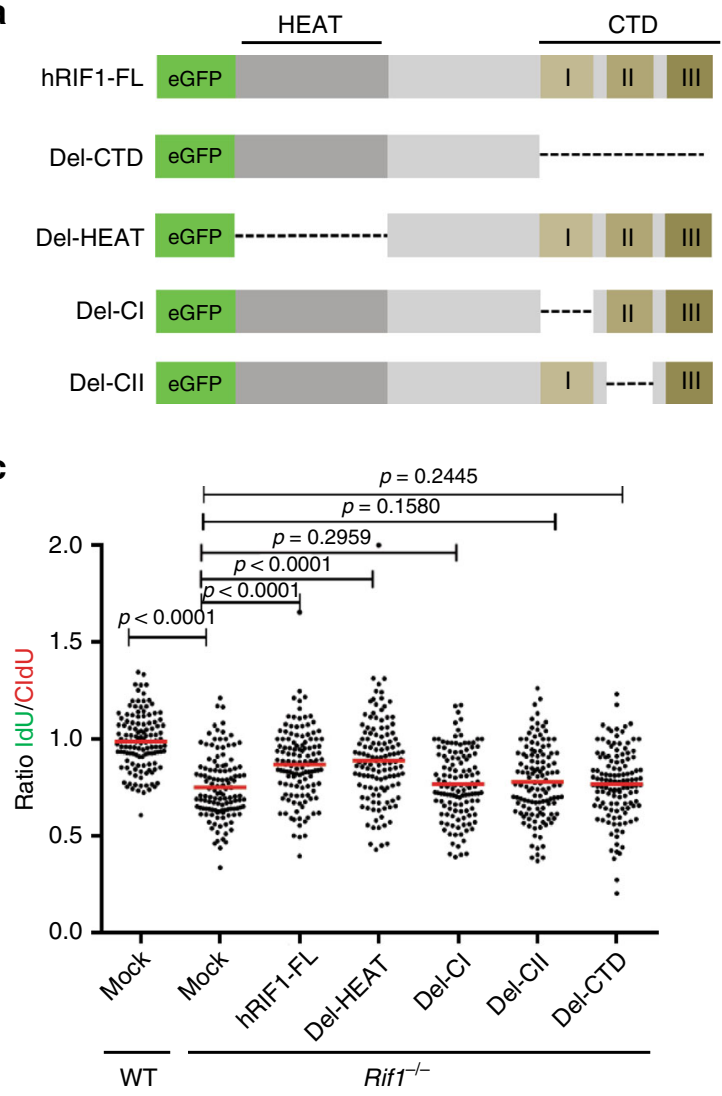

e

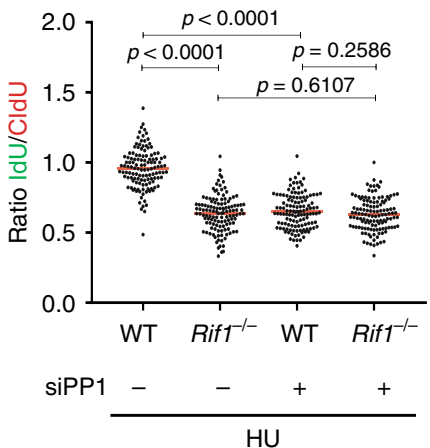

f

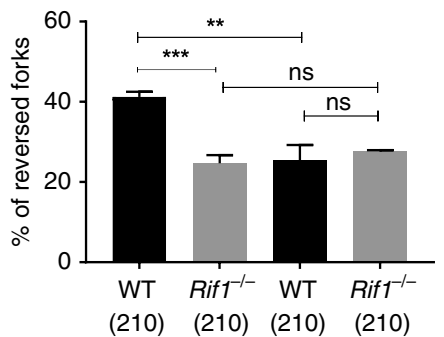

PP1i

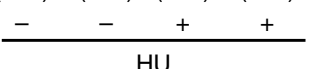

b

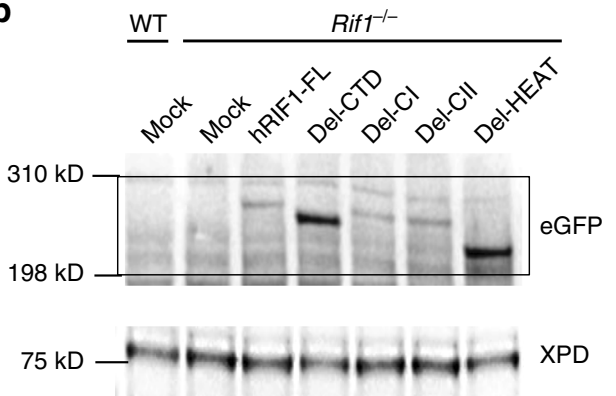

d

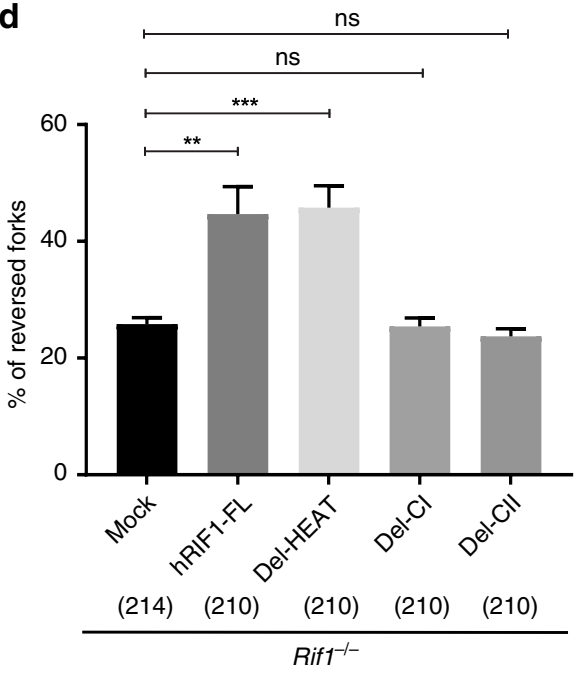

g
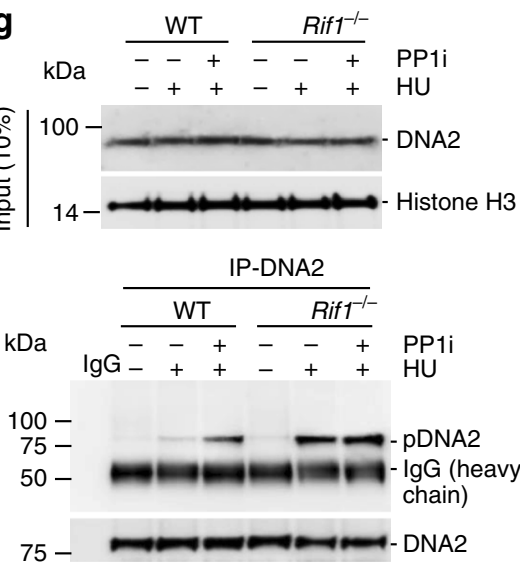

Fig. 4 C-terminal region of RIF1 protects of reversed forks from degradation. a Schematic of full-length (FL) human RIF1 protein and deletion mutant constructs. Deleted region for each mutant is denoted by dotted line. b Western blot analysis for Rif1- I- MEFs transfected with mutant construct of human RIF1. Lysates were probed with antibody against GFP. XPD was used as loading control. Expression of mutant protein is visualized as distinct bands in range of $198 \mathrm{kD}$ to $310 \mathrm{kD}$, which is missing in mock-transfected samples. c DNA fiber assay to assess the rescue of Fork degradation in Rif1-/- MEFs transfected with RIF1 mutant constructs (for $48 \mathrm{~h}$ ) upon treatment with $4 \mathrm{mM} \mathrm{HU}$ for $3 \mathrm{~h}$. d Percentage of fork reversal in Rif1-/- MEFs transfected with different mutant constructs of human RIF1 and subsequent treatment with $\mathrm{HU}$ for $3 \mathrm{~h}(4 \mathrm{mM})$. Numbers of analyzed molecules are indicated in parentheses. e DNA fiber assay to determine the extent of fork degradation in WT and Rif1 ${ }^{-/}$MEFs upon siRNA-mediated downregulation of PP1. $\mathbf{f}$ Percentage of reversed forks observed in WT and Rif1-/- MEFs treated with $4 \mathrm{mM} \mathrm{HU}$ for $3 \mathrm{~h}$ with or without inhibiting PP1. Number of molecules analyzed are indicated within the parenthesis. At least 125 readings were taken for $\mathbf{c}$ and $\mathbf{e}$ and the mean values are represented by red bar. $P$-values were derived from Kruskal-Wallis ANOVA with Benjamini Hochberg post test for $\mathbf{c}$ and $\mathbf{e}$ and from unpaired $t$-test for $\mathbf{d}$ (ns, non-significant, ${ }^{\star \star \star} P=0.0009$, $\left.{ }^{\star \star} P=0.0025\right)$ and $\mathbf{f}$ (ns, nonsignificant, ${ }^{\star \star}{ }^{\star} P=0.0003,{ }^{\star \star} P=0.0026$ ). All the experiments were repeated for three times with similar outcomes (Supplementary Data 2 and Supplementary Fig. 8a, b). $\mathbf{g}$ DNA2 is hyper phosphorylated in Rif1-/- MEFs during replication stress. Top panel: level of DNA2 in nuclear extracts from WT and Rif1 ${ }^{-1-}$ MEFs before and after treatment with $\mathrm{HU}$ alone or in combination with PP1 inhibitor treatment (tautomycetin $225 \mathrm{nM}$ for $2 \mathrm{~h}$ ). Western blots were performed with antibody against DNA2 antibody. Histone H3 was used as loading control. Bottom panel: Immunoprecipitations were carried out with anti-DNA2 antibody or the corresponding $\lg \mathrm{G}$ and were probed with $\mathrm{p}-(\mathrm{S} / \mathrm{T})$ antibody 
inhibitor tautomycetin (PP1i) ${ }^{44,45}$ resulted in similar degradation profiles as observed upon knockdown of PP1 (Supplementary Figs. 4d and 8c). HU treatment after PP1 inhibition in WT cells also resulted in a significant decrease of fork reversal frequencies (Fig. 4f and Supplementary Data 3). PP1i treatment in RIF1deficient cells did not further decrease the amount of fork reversal to levels observed in either Rif1 $1^{-1-}$ cells or WT cells treated PP1i (Fig. 4f), suggesting that RIF1 and PP1 are epistatic for preventing the degradation of reversed forks (Fig. 4e, f, Supplementary Fig. 8b, c and Supplementary Data 3).

DNA2 phosphorylation was shown to be important for recruitment to DSBs in yeast ${ }^{46}$. We, therefore, hypothesized that access of DNA2 to forks upon replication stress could be controlled by PP1 in a phosphorylation-dependent manner. To test this hypothesis, we immunoprecipitated DNA2 from nuclear extracts of WT or Rif1-/- MEFs treated with either HU or HU and PP1i. The immunoprecipitated DNA2 was then probed for phosphorylation status using the phospho-S/TQ motif antibody. Treatment with HU slightly increased the levels of DNA2 phosphorylation in WT cells when compared to untreated cells (Fig. 4g). PP1 inhibition markedly increased the phosphorylation levels of DNA2 upon HU treatment (Fig. 4g). Additionally, DNA2 phosphorylation levels in RIF1-deficient cells upon HU treatments were observed to be similar to WT cells upon PP1 inhibition. The DNA2 phosphorylation status was not further increased upon inhibition of PP1 in RIF1-deficient cells upon HU treatment, suggesting that RIF1-PP1 interaction controls DNA2 phosphorylation levels upon replicative stress (Fig. $4 \mathrm{~g}$ ).

RIF1 deficiency results in defective fork restart. Nascent strand degradation has been linked to increased genome instability ${ }^{4}$. We, therefore, tested if fork degradation in RIF1-deficient cells induces immediate induction of DSBs. We performed pulsed-field gel electrophoresis (PFGE) analysis ${ }^{16}$ where we did not observe a significant difference between WT and Rif1-l- MEFs (Fig. 5a, b). Treatment with HU resulted in a marginal but non-significant increase of DSBs both in WT and Rif1-I- cells, when compared to their non-treated counterparts (Fig. 5a, b). These low levels of DSBs observed in RIF1-deficient cells were not suggestive of fork collapse into DSBs upon degradation, a phenomenon that was observed on the entire population of active forks (3000-12,000 per cell) ${ }^{47}$ (Fig. 5b).

We next tested if fork degradation resulted in genome instability in WT and Rif $1^{-1-}$ MEFs treated with replication stress-inducing agents HU, cisplatin, and Camptothecin (CPT) (Fig. 5c, d and Supplementary Fig. 5a) by metaphase spreads. Untreated Rif1 ${ }^{-1-}$ cells did not show a significant increase in aberrant chromosomes (Fig. 5c, d and Supplementary Fig. 5a). However, upon HU, cisplatin or CPT treatment, Rif1-l- MEFs displayed significantly increased aberrations when compared to their WT counterparts (Fig. 5c, d and Supplementary Fig. 5a). Furthermore, consistent with previous data 27,48 , clonogenic survival assays performed in WT and Rif1 ${ }^{-1-}$ MEFs showed that RIF1 deficiency also resulted in increased sensitivity to HU, cisplatin or CPT (Fig. 5e, f and Supplementary Fig. 5b). These data show that although fork degradation does not result in immediate replication fork collapse, it results in increased genome instability and sensitivity to replication stress.

We hypothesized that the increased genome instability in RIF1-deficient cells could arise from defective restart of stalled replication forks. To test this, we performed a fork restart assay, in which cells were labeled with CldU followed by HU treatment to stall the forks and then released into IdU (Fig. 5g). However, WT and RIF1-deficient cells did not reveal a significant difference between stalled versus restarted forks, suggesting that the majority of forks were restarted (Supplementary Fig. 5c). Further analysis of individual tract lengths revealed that restarted forks from Rif $1^{-1-}$ cells showed significantly shorter IdU tracts, suggestive of a delayed restart in these cells (Fig. 5h and Supplementary Fig. 8d). A similar trend of delayed fork restart was also observed in RIF1-KO HAP1 cells (Supplementary Figs. $5 \mathrm{~d}$ and $8 \mathrm{e}$ ). Shorter inter-origin distances could also account for smaller IdU labels in RIF1-deficient cells upon restart. To test this, we allowed the forks to restart after HU treatments for multiple time points ranging from $15^{\prime}$ to $60^{\prime}$. A significant decrease in the percentage of restarted forks was observed at early time points after release $\left(15^{\prime}\right.$ and $\left.30^{\prime}\right)$ in Rif1 $1^{-/-}$cells, but not at later time points $\left(45^{\prime}\right.$ and $\left.60^{\prime}\right)$ (Supplementary Fig. 5e). However, the CldU tract lengths at $30^{\prime}, 45^{\prime}$, and $1 \mathrm{~h}$ show significant shorter tracts in RIF1-deficient cells, suggesting that the shorter tracts could be due to delayed restart in these cells (Supplementary Figs. $5 \mathrm{f}$ and $8 \mathrm{f}$ ).

Since 53BP1 was recently implicated in replication fork restart ${ }^{49}$, we wondered if the restart defect observed upon RIF1 inactivation is epistatic with 53BP1. To this end, we used 53BP1 $15 \mathrm{~A}$ MEFs, which lack 15S/TQ phosphorylation sites within 53BP1 essential for RIF1 binding ${ }^{26}$. 53BP $1^{15 \mathrm{~A}}$ cells did not display a defect, suggesting that RIF1 and 53BP1-mediated restart is differentially regulated (Supplementary Figs. $5 g$ and $8 \mathrm{~g}$ ). These data suggested that the genome instability and sensitivity observed in RIF1-deficient cells could be a result of defective restart in these cells.

Restart delay results in genome instability. To explore whether fork restart defects in RIF1-deficient cells cause genome instability, we tested directly if forks restarted after HU treatments resulted in formation of DSBs. WT and Rif1-l- MEFs were assayed for formation of DSBs by PFGE at $15 \mathrm{~h}$ after release from $\mathrm{HU}$-induced fork stalling. As observed previously, HU treatment in either WT or RIF1-deficient cells did not cause a significant change in DSBs frequency (Figs. 5a, b and 6a, b). However, Rif1- ${ }^{-}$cells displayed a significant increase of DSBs compared to WT cells (Fig. 6a, b), which could be a result of decreased repair of DSBs after release. Since RIF1-deficient do not have a HR defect, we also tested if these cells show defective NHEJ. Using a reporter-based NHEJ assay ${ }^{50}$, we found a significant decrease in NHEJ levels in RIF1-deficient cells when compared to WT cells, consistent with published evidence ${ }^{17}$ (Supplementary Fig. 6a). Since the 53BP1-RIF1 axis is responsible for NHEJ repair, we next tested if 53BP1 deficiency also resulted in genome instability upon replication stress. In contrast to RIF1deficient cells, which showed high levels of genome instability, 53BP1 deficiency did not result in significant chromosomal aberrations upon either $\mathrm{HU}$ or cisplatin treatments (Supplementary Fig. 6b). Taken together, these data suggest that defective NHEJ-mediated repair could be involved in increased DSB formation in RIF1-deficient cells upon restart. However, this cannot completely account for the increased genome instability observed in RIF1-deficient cells, as 53bp $1^{-1-}$ MEFs did not show increased levels of genome instability upon induction of replication stress.

To further test if the delayed restart resulted in increased singlestranded DNA (ssDNA) levels in these cells, we analyzed RPA2, a surrogate for ssDNA, by flow cytometry. Upon HU treatment, the replication-associated RPA2 signals were markedly enhanced in both Rif1 $1^{-1-}$ and WT cells (Supplementary Fig. 6c, d). At 5 h after release from a HU-mediated block, slightly reduced but still significantly higher levels of RPA were observed in both the cell types (Supplementary Fig. 6c, d). However, at $15 \mathrm{~h}$ after $\mathrm{HU}$ release, WT cells showed low RPA levels, along with normal cell cycle profiles. In contrast, Rif1 $1^{-1-}$ cells displayed an accumulation 
a

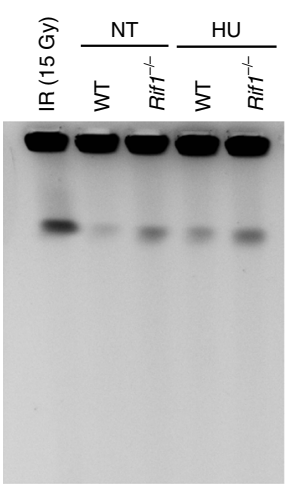

c

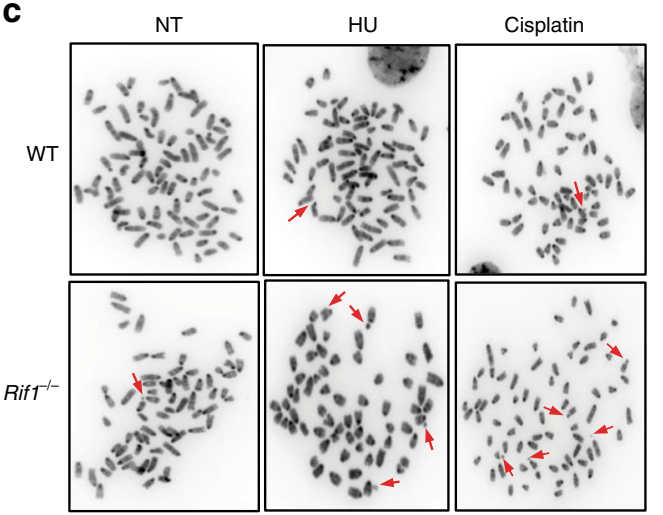

e
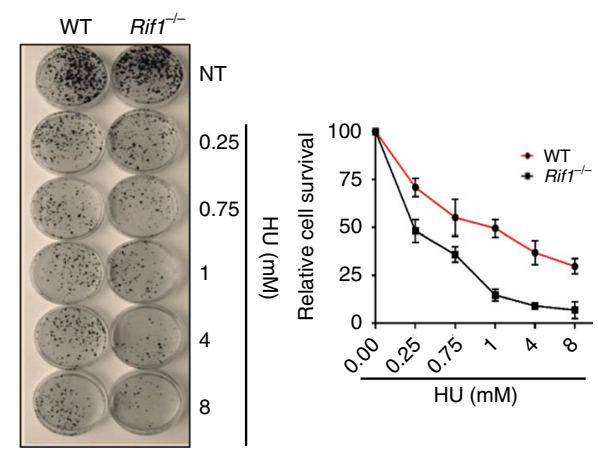

g

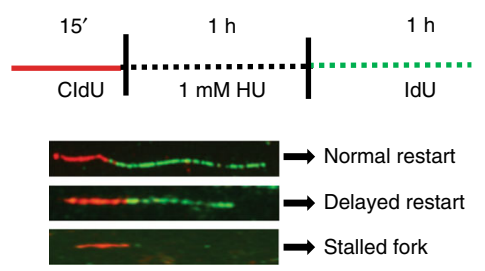

of cells in late S/G2 with significantly higher percentages of RPA2positive cells (Supplementary Fig. 6c-e). To subsequently test if RIF1-deficient cells entered mitosis with high levels of underreplicated DNA, we performed co-staining for phospho-histone H3 in combination with RPA2 using the same experimental conditions as in Supplementary Fig. 6c. However, we did not observe any significant differences in phospho-histone H3-positive cells between the two genotypes (Supplementary Fig. 6f), suggesting that upon restart, RIF1-deficient cells expose increased

b

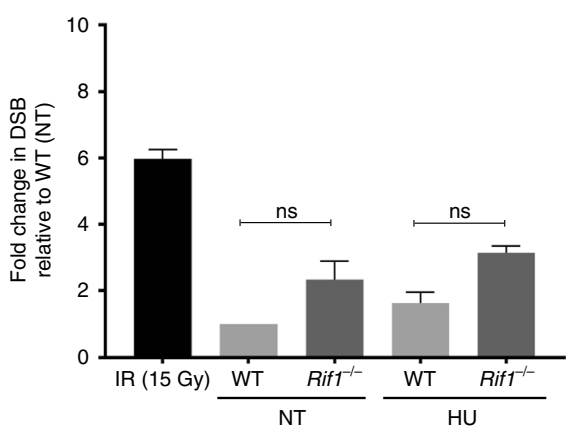

d

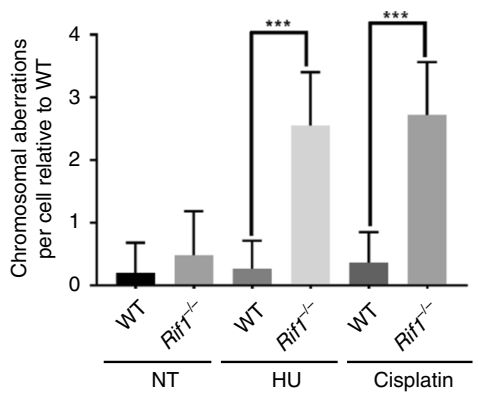

f

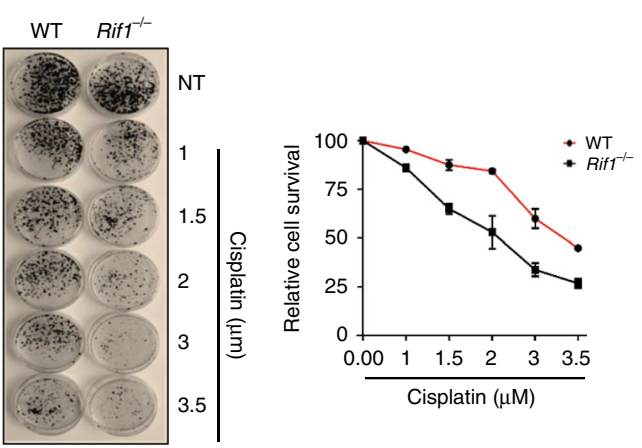

h

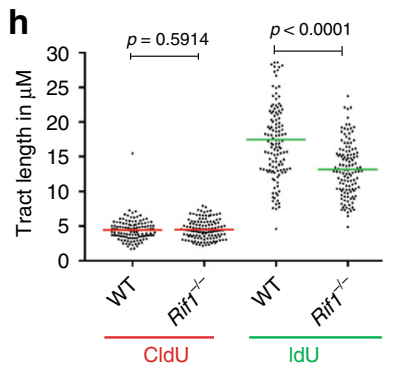

amounts of ssDNA, which causes accumulation in late S/G2 phase of the cell cycle.

We speculated that the increased levels of ssDNA in RIF1deficient cells could be a result of under-replicated DNA during the restart process. To test this hypothesis, we performed EM analysis of restarted forks. Interestingly, we observed a significant increase in replication intermediates with high levels of ssDNA at forks in RIF1-deficient cells when compared to WT cells (Fig. 6c, e). Furthermore, a significant increase was observed in ssDNA gaps 
Fig. 5 Delayed fork restart and genomic instability observed upon RIF1 deficiency. a PFGE analysis for DSBs in WT and Rif1-/- MEFs with and without treatment with HU for $3 \mathrm{~h}$. WT MEFs treated with IR (15 Gy) was taken as positive control. b Quantification of experiment (a), an integration of three independent experiments showing DSB levels relative to WT untreated (NT), (ns, not-significant, from unpaired $t$-test). c Representative images for analysis of genomic instability analysis by metaphase spread in WT and Rif1-/- MEFs upon HU and Cisplatin treatment. d Quantitation of chromosomal aberrations in c. Sixty metaphase fields per conditions were analyzed and three independent experiments were carried out. $P$-value was calculated by unpaired $t$-test $\left({ }^{\star \star \star} P \leq 0.0001\right)$. e-f Images for clonogenic survival assay in WT and Rif1-/- MEFs treated with different concentrations of HU (e) and Cisplatin (f) after which the drugs were washed off and the cells were allowed to grow for 8 days. Adjoining graphs show the data from three independent experiments. Error bars represent s.e.m. $\mathbf{g}$ Schematics of fork restart assay by DNA fibers and representative images for normal restart, delayed restart and stalled fork upon release from HU treatment. $\mathbf{h}$ Quantitation for restart assay in $\mathbf{g}$. Tract lengths of IdU and CldU were quantified in WT and Rif1-/- MEFs upon restart after treatment with $1 \mathrm{mM} \mathrm{HU}$ for $1 \mathrm{~h}$ from 125 fibers per sample. Red and green bars indicate mean CldU and IdU tract length. $P$-values were derived from Kruskal-Wallis ANOVA with Benjamini Hochberg post test. All experiments were repeated three times (Supplementary Data 2 and Supplementary Fig. 8d)

behind forks in Rif1 ${ }^{-1-}$ cells (Fig. 6d, f). To test if ssDNA regions observed in RIF1-deficient cells were a result of the fork degradation process, we inhibited DNA2 in WT and Rif $1^{-1-}$ cells before release from $\mathrm{HU}$ block. Interestingly, DNA2 inhibition in RIF1-deficient cells significantly reduced both the ssDNA regions at the forks and the gaps behind the forks in RIF1-deficient cells (Fig. 6e, f). These data suggest that the increased ssDNA regions observed at and behind the replication forks in RIF1-deficient cells could be a consequence of defective restart caused due to fork degradation. To verify this hypothesis, we performed a fork restart assay, in which WT and Rif1-l- cells were pre-incubated with DNA2i during HU treatment to prevent fork degradation. Forks were then allowed to restart, and subsequently assessed for IdU tract length (Fig. 6g). DNA2 inhibition during $\mathrm{HU}$ treatment completely rescued the restart delay in RIF1-deficient cells (Fig. $6 \mathrm{~g}$ and Supplementary Fig. 8h). Furthermore, complementation of Rif1 $1^{-1-}$ MEFs with hRIF1-FL or the Del-HEAT mutant rescued the restart defect in RIF1-deficient cells (Fig. 6h and Supplementary Fig. 8i). However, complementation with either Del-CI or DelCII did not restore the restart defect upon RIF1 deficiency, in good agreement with our earlier data that these domains are essential for protection of reversed forks (Figs. $6 \mathrm{~h}$ and $4 \mathrm{c}, \mathrm{d}$ ). These data further strengthen the concept that protection of reversed forks from degradation is linked to efficient fork restart.

Finally, we tested if allowing efficient restart in RIF1-deficient cells could rescue the observed sensitivity to replication stressinducing agents. Rif1-1- MEFs were complemented with either hRIF1-FL, Del-HEAT, Del-CI or Del-CII, and treated with either HU or cisplatin. Complementation of Rif1 $1^{-1-}$ with either hRIF1FL or Del-HEAT significantly rescued the sensitivity of cells to replication stress, in line with our molecular data (Fig. $6 \mathrm{i}$ and Supplementary Fig. 6g). In contrast, complementation with DelCI or Del-CII failed to rescue the sensitivity of RIF1-deficient cells (Fig. 6i and Supplementary Fig. 6g). These results strongly suggest that replication fork protection and subsequent efficient fork restart are physiologically important processes for cellular survival in situations of replication stress.

\section{Discussion}

Our findings identify a novel role of RIF1 in the protection of nascent strands, which underpins how degradation of reversed replication forks can result in genome instability. Replication fork degradation results in genome instability in HR- and FA-defective cells $^{3,4}$. However, it remained poorly understood how degradation of reversed forks results in genome instability.

We show that RIF1 associates with stalled forks and protects them from DNA2-mediated degradation, which is independent of its known interaction with 53BP1 $1^{17,19-21,25,26}$ and thus NHEJ (Figs. 1-3). We further show that loss of Rif1 results in deprotection of reversed forks, resulting in extensive fork degradation (Fig. 2b-f). Importantly, RIF1 was found to act downstream of RAD51, which is involved in fork reversal and in protection of regressed arms (Fig. $2 \mathrm{~g}, \mathrm{~h}$ ).

Recent studies have also implicated reversed replication forks to be a substrate for MRE11 nuclease action in BRCA2-deficient cells. Other nucleases, including DNA2, MUS81, and EXO1, have also been proposed to mediate fork degradation ${ }^{7,8,51}$. Degradation of reversed forks upon RIF1 deficiency appears to be primarily dependent on DNA2 activity, with a partial requirement of MRE11. These findings suggest that whereas MRE11 can partially access the reversed arm upon RIF1 deficiency, DNA2 is the main nuclease in the degradation process in these conditions (Figs. 2 and 3).

Our data also show that the C-terminal region of RIF1 (consisting of sub-domains CI, CII, and CIII) is essential for protecting reversed forks from degradation (Fig. 4c, d). The CI region has two conserved binding sites for PP $1 a^{40}$, CII region binds to cruciform DNA structures ${ }^{43}$, while the complete C-terminal domain is responsible for BLM binding ${ }^{48}$. Our data demonstrate that the CII domain of RIF1, which binds to cruciform structures, is critical for the protection of reversed forks upon replication stress (Fig. 4c, d). One possibility could be that RIF1 binds to reversed forks, which represent cruciform structures in vivo upon replication stress, and physically protect such forks. Another possibility involves the requirement of both the functions of $\mathrm{CI}$ and CII domains of RIF1 in fork protection, as also suggested by our data (Fig. 4c-e). In this scenario, binding of the CII domain to reversed forks could then recruit $\mathrm{PP} 1$ through the $\mathrm{CI}$ domain to the forks. This recruitment of PP1 could post-translationally restrict DNA2 nuclease activity though de-phosphorylation of DNA2 in the vicinity of forks, thereby protecting them from degradation. In line with this hypothesis, we show that downregulation/ inhibition of PP1 results in reversed fork degradation in WT cells, in a fashion that is epistatic with RIF1 inactivation (Fig. 4e, f). Furthermore, RIF1 inactivation results in hyper-phosphorylation of DNA2 upon replication stress, which again is epistatic with inhibition of PP1 (Fig. $4 \mathrm{~g}$ ). Therefore, one could envision a scenario where access of DNA2 to stalled forks is fine-tuned through PP1-mediated phosphorylation/de-phosphorylation cycles to prevent unrestricted processing of stalled replication forks.

Importantly, our data also provide insight into the mechanisms by which reversed fork degradation results in genome instability. We show that fork degradation upon RIF1 deficiency causes delayed restart, which could be the precursor for subsequent genome instability (Figs. 5 and 6a). RIF1 has multiple roles in the maintenance of genome stability, including in the regulation of origin firing and also in NHEJ. Although disruption of these processes could also contribute to genome instability, our data strongly suggest that delayed restart and subsequent exposure of ssDNA could also contribute to the genome instability upon loss of RIF1 (Figs. 5 and 6). 
a

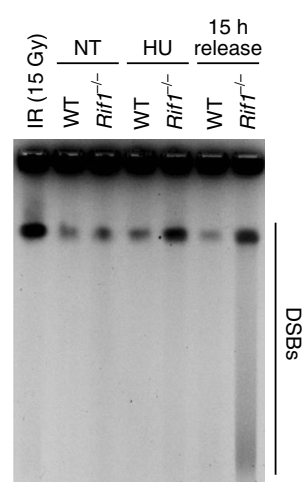

C

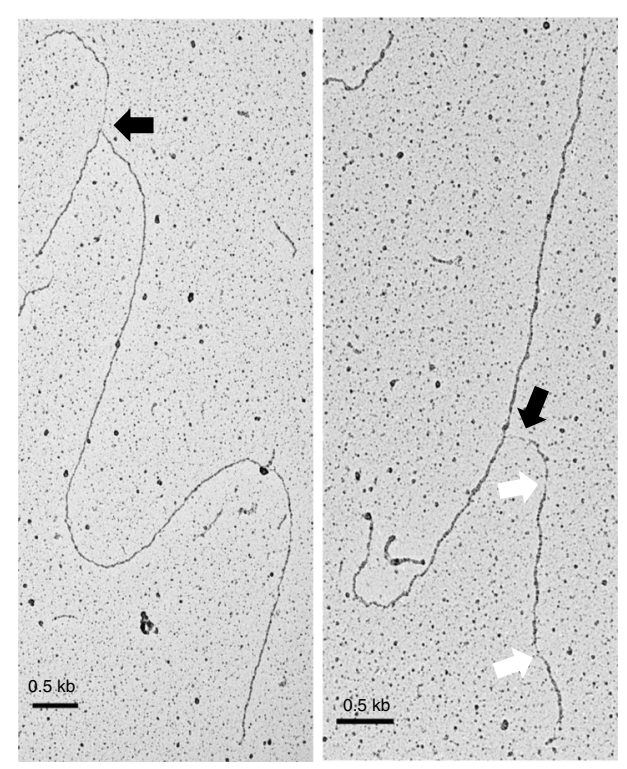

h

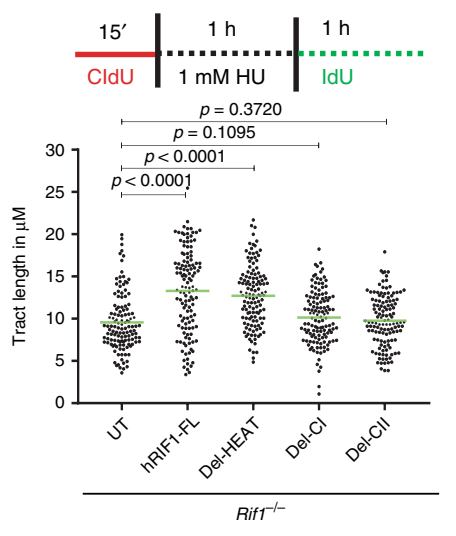

i b

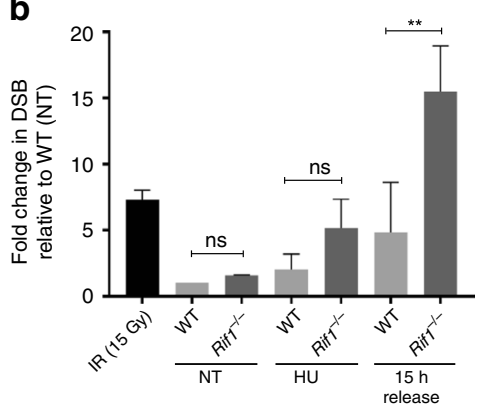

e

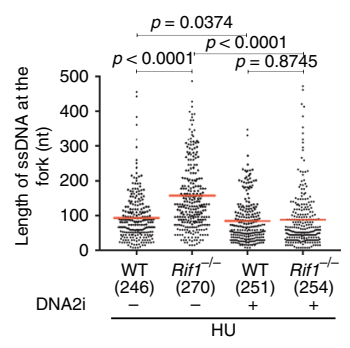

f

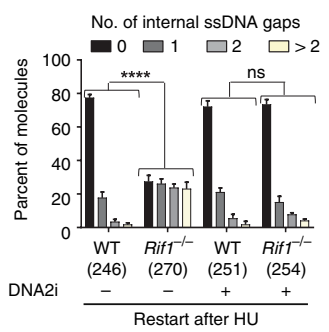

g
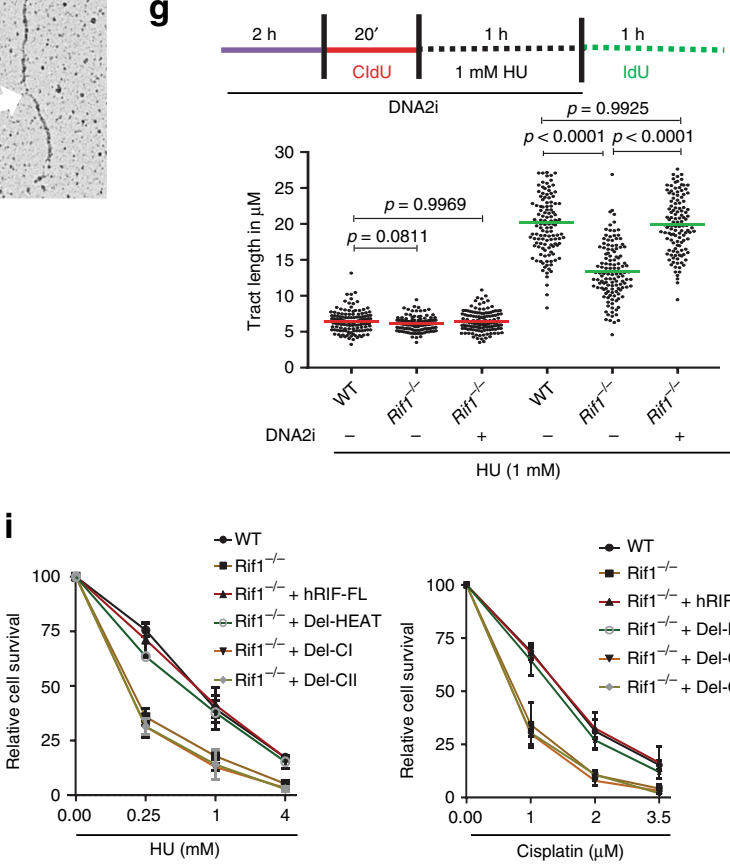

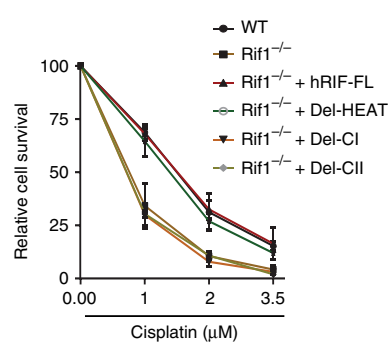

Restart of reversed forks can take place via multiple nonmutually exclusive mechanisms. One mechanism includes helicase-mediated branch migration of the "reversed arm" by RecQ1 helicase ${ }^{52}$. However, upon fork degradation, cells can employ alternate pathways for restart. One such pathway involves re-priming events ahead of the stalled forks. However, re-priming can result in gaps in the daughter strands ${ }^{46,53}$. In line with this speculation, RIF1-deficient cells accumulate increased ssDNA gaps behind the forks when allowed to restart after replication stress. Notably, this phenomenon was dependent on DNA2 activity (Fig. 6c-e). Furthermore, our data indicate that prevention of reversed fork degradation rescues the defective restart in RIF1-deficient cells (Fig. 6f-h). We propose a model, in which RIF1 protects reversed forks from degradation and mediates 
Fig. 6 Restart defects are a consequence of fork degradation in RIF1-deficient cells. a PFGE in WT and Rif1-/- MEFs with and without treatment with HU for $3 \mathrm{~h}$ and $15 \mathrm{~h}$ recovery after treatment. b Quantification of experiment (a), from three independent experiments showing DSB levels relative to WT untreated (NT), (ns, not-significant, ${ }^{\star \star} P=0.0019$, unpaired $t$-test). c, $\mathbf{d}$ Electron micrographs of ssDNA at the fork (c), and behind the fork (d), 30 min after release from HU treatment. White arrows represent ssDNA at the forks and black arrows in d, represent ssDNA gaps behind the forks e Analysis of ssDNA at forks upon restart in WT and Rif1-/- MEFs in presence or absence of DNA2 inhibitor. Red bar represents mean, $P$-value was derived from Kruskal-Wallis ANOVA with Benjamini Hochberg post test. f Analysis of internal gaps behind forks upon restart in WT and Rif1-/- MEFs in the presence or absence of DNA2 inhibitor and HU. Graph represents mean and SD from three independent experiments. Chi-square test of trends was done to assess significance of internal ssDNA gaps between WT and Rif1 ${ }^{-/}-$MEFs (ns, non-significant, ${ }^{\star \star \star \star} P<0.0001$ ). Numbers of analyzed molecules are indicated within parenthesis for e, f. g Top: schematics for restart assay by fibers upon DNA2 inhibition. Bottom: Tract lengths of IdU and CldU were quantified in WT and Rif1-/- MEFs upon restart after treatment with $1 \mathrm{mM} \mathrm{HU}$ for $1 \mathrm{~h}$ in the presence or absence of DNA2i. $\mathbf{h}$ Top: schematics for fiber restart assay upon transfection of hRIF1 deletion mutant constructs in Rif1 ${ }^{-/}$MEFs. Bottom: Quantification of IdU tracts in Rif1-/- MEFs upon restart after treatment with $1 \mathrm{mM} \mathrm{HU}$ for $1 \mathrm{~h}$ in the presence or absence of hRIF1 deletion constructs. Red and green bars in $\mathbf{g}$ and $\mathbf{h}$ represents mean CldU and IdU tract length, $P$ values were obtained from Kruskal-Wallis ANOVA with Benjamini Hochberg post test for FDR. All experiments were repeated thrice (Supplementary Data 2 and Supplementary Fig. 8h-i). i Survival assay in Rif1 ${ }^{-/-}$MEFs complemented with hRIF1-FL, Del-HEAT, Del-Cl, Del-CII constructs of hRIF1 and treated with different concentrations of $\mathrm{HU}$ and Cisplatin. Data represented from three independent experiments, error bars represent s.e.m

efficient restart due to the presence of the reversed arm as substrate for branch migration (Fig. 7a, b). Absence of RIF1 leads to extensive fork degradation, resulting in delayed restart. This delayed fork restart results in the exposure of under-replicated DNA behind the forks (Fig. 7c). The under-replicated DNA then becomes a source of genome instability later (Fig. 7c).

Identification of the mechanisms underlying replication fork degradation is also clinically relevant, as fork protection in BRCA-deficient tumors has recently been implicated in chemoresistance ${ }^{5,6}$. We speculate that fork degradation at difficult-to-replicate regions of the genome could be a potential source of genome instability. Consistent with this idea, RIF1deficient cells show a slightly higher background level of genome instability (Fig. 5a). These low -but tolerable- levels of genome instability combined with a checkpoint defect could result in accelerated tumorigenesis. On the other hand, cancers with RIF1 mutations could be more responsive to chemotherapeutic regimens. Although further studies are required to test these hypotheses, mechanistic insights into the process of replication fork protection could result in the development of potentially new therapeutic regimens for cancer.

\section{Methods}

Cell culture, cell lines, and transfection reagents. All the MEFs (WT, Rif1 $1^{-1-}$, $53 b p 1^{-l-}$, and 53BP1 $\left.1^{15 A}\right)^{30}$ were cultured in Dulbecco's Modified Eagle Medium (DMEM) supplemented with $10 \%$ fetal calf serum (FCS) and $1 \%$ penicillin-streptomycin (PS, P0728 Sigma) at $37^{\circ} \mathrm{C}$ and $5 \%$ in a humidified incubator. Transfections were performed using transfection reagents Xtremegene- 9 (Roche) and Lipofectamine-2000 according to the manufacturer's protocol. WT and RIF1-KO HAP1 ${ }^{27}$ were cultured in Iscove's Modified Dulbecco's Media (IMDM) containing $10 \%$ FCS and $1 \%$ pen-strep.

Generation of deletion mutants. RIF1 mutants were created using the standard PCR and cloning methods. The following primers were used for creating the deletion mutants for various domains of human RIF1:

hRif-DelCTD-Rev : 5'-GACACAGCGTGTCTGCA-3'

hRif-DelCTD-Fwd : 5'-TAGGACCCAGCTTTCTTGTAC-3'

hRif-DelHEAT- Rev: 5' - CATGGTGAAGCCTGCT-3'

hRif-DelHEAT-Fwd: 5' - CCTGGTTTGGAAACTGTTGAAAT-3

hRif-DelC1-Fwd: 5'-CAATCTAAGATTTCAGAAATGGCCA-3'

hRif-DelC2-Rev: 5' - GTTCACCAATGGTGGGTAAACA -3'

hRif-DelC2-Fwd: 5' - CTAGAAGAGATTCCAGTTTTTGATATTTCT -3'

The GFP-RIF1 constructs used in this study is based on pcDNA5/FRT/TOGFP-RIF1 described previously ${ }^{24}$, which has human RIF1 cDNA fused to GFP at its N-terminus. Domain deletions were created using Q5 Mutagenesis Kit (NEB, cat. No\# E0554S), following the manufacturer's instruction. Primers were used to PCR amplify the entire plasmid leaving out the region of RIF1 to be deleted. PCR products were gel purified and ligated. Introduction of domain deletions were further verified by Sanger sequencing.

iPOND-SILAC mass-spectrometry. For SILAC labeling, mouse embryonic cells (mESCs) were maintained in serum free $2 \mathrm{i}$ media deficient in lysine, arginine, and
L-glutamine (PAA) at $37^{\circ} \mathrm{C}$ and $5 \% \mathrm{CO} 2$ in a humidified incubator. Cells were grown in medium containing either $73 \mu \mathrm{g} / \mathrm{ml}$ light $\left[{ }^{12} \mathrm{C}_{6}\right]$-lysine and $42 \mu \mathrm{g} / \mathrm{ml}$ $\left[{ }^{12} \mathrm{C}_{6},{ }^{14} \mathrm{~N} 4\right]$-arginine (Sigma) or similar concentrations of heavy $\left[{ }^{13} \mathrm{C}_{6}\right]$-lysine or $\left[{ }^{13} \mathrm{C}_{6},{ }^{15}{ }_{\mathrm{N} 2}\right]$-lysine and or $\left[{ }^{13} \mathrm{C}_{6},{ }^{15}{ }_{\mathrm{N} 4}\right]$-lysine arginine (Cambridge Isotope Laboratories).

For iPOND experiments, cells were labeled with $10 \mu \mathrm{M}$ EdU for $10 \mathrm{~min}$ and then treated with $\mathrm{HU}(4 \mathrm{mM})$ for $2 \mathrm{~h}$ to stall the DNA replication forks. After labeling and treatment cells were washed with Phosphate Buffer Saline (PBS) and harvested using cell scrapper. Samples were then treated with click reaction containing $25 \mu \mathrm{M}$ biotin-azide, $10 \mathrm{mM}(+)$ sodium L-ascorbate and 2 $\mathrm{mM} \mathrm{CuSO}_{4}$ and rotated at $4{ }^{\circ} \mathrm{C}$ for $1 \mathrm{~h}$. Samples were then centrifuged to pellet down the cells; supernatant was removed and replaced with $1 \mathrm{ml}$ Buffer-1 containing $25 \mathrm{mM} \mathrm{NaCl}, 2 \mathrm{mM}$ EDTA, $50 \mathrm{mM}$ Tris- $\mathrm{HCl}$, pH 8.0, $1 \%$ IGEPAL and protease inhibitor and rotated again at $4{ }^{\circ} \mathrm{C}$ for $30 \mathrm{~min}$ This step was repeated twice. Samples were centrifuged to pellet down the cells; supernatant was removed and replaced with $500 \mu \mathrm{l}$ of B1 and sonicated 30 times for $20 \mathrm{~s}$ on and $90 \mathrm{~s}$ off at high amplitudes using a Diagenode Bioruptor plus sonicator. Samples were centrifuged, and supernatant was transferred to fresh tubes and incubated for $1 \mathrm{~h}$ with $200 \mu \mathrm{l}$ of Dyna-Beads My-One C1 for the streptavidin biotin capture step. Proteins were eluted, and mass-spectrometry was performed. At least two peptides were required for protein identification. Quantitation is reported as the $\log _{2}$ of the normalized heavy/light ratios. SILAC data were analyzed using MaxQuant. The resulting output tables of two independent experiment were merged and used as the input for calculating the average fold-change to identify significantly upregulated proteins upon $\mathrm{HU}$ treatment based on H:L ratio in the SILAC experiment in the MaxQuant software ${ }^{54}$.

Immunoblotting. Cells were lysed in $4 \mathrm{x}$ Laemmli sample buffer and boiled for 5 min. Proteins were separated on $4-12 \%$ NuPAGE Bis-Tris Gel (Novex life technologies) and transferred on nitrocellulose membrane $(0.45 \mu \mathrm{M})$. Membranes were blocked with $5 \%$ milk in PBS-1\% Tween 20 for $1 \mathrm{~h}$ and incubated overnight in primary antibodies. Membranes were then washed three times with PBS containing $0.05 \%$ tween and probed with respective secondary antibodies. Finally ECL Prime Western Blotting Detection Reagent kit (GE Healthcare) was used to develop the blots. Details of the antibodies used are provided in Supplementary Table 1 .

DNA fiber analysis. DNA fiber analysis was carried out according to the standard protocol as mentioned previously ${ }^{18}$. Briefly, cells were sequentially pulse-labeled with $30 \mu \mathrm{M}$ CldU (c6891, Sigma-Aldrich) and $250 \mu \mathrm{M}$ IdU (I0050000, European Pharmacopoeia) for $20 \mathrm{~min}$ and treated with $\mathrm{HU}(4 \mathrm{mM})$ for $3 \mathrm{~h}$ for fork degradation assay, and for fork restart assay after first labeling with CldU cells were treated with $1 \mathrm{mM} \mathrm{HU}$ for $1 \mathrm{~h}$. After labeling, cells were collected and resuspended in PBS at $2.5 \times 10^{5}$ cells per ml. The labeled cells were mixed at $1: 1(\mathrm{v} / \mathrm{v})$ with unlabeled cells, and $2.5 \mu \mathrm{l}$ of cells were added to $7.5 \mu \mathrm{l}$ of lysis buffer $(200 \mathrm{mM}$ Tris$\mathrm{HCl}, \mathrm{pH} 7.5,50 \mathrm{mM}$ EDTA, and $0.5 \%(\mathrm{w} / \mathrm{v}) \mathrm{SDS}$ ) on a glass slide. After $8 \mathrm{~min}$, the slides were tilted at $15-45^{\circ}$, and the resulting DNA spreads were air dried, fixed in $3: 1 \mathrm{methanol} /$ acetic acid overnight at $4^{\circ} \mathrm{C}$. The fibers were denatured with $2.5 \mathrm{M}$ $\mathrm{HCl}$ for $1 \mathrm{~h}$, washed with PBS and blocked with $0.2 \%$ Tween 20 in $1 \%$ BSA/PBS for $40 \mathrm{~min}$ The newly replicated CldU and IdU tracks were labeled (for $2.5 \mathrm{~h}$ in the dark, at room temperature (RT)) with anti-BrdU antibodies recognizing CldU (1:500, ab6326; Abcam) and IdU (1:100, B44, 347580; BD), followed by $1 \mathrm{~h}$ incubation with secondary antibodies at RT in the dark: anti-mouse Alexa Fluor 488 (1:300, A11001, Invitrogen) and anti-rat Cy3 (1:150, 712-166-153, Jackson Immuno-Research Laboratories, Inc.). Fibers were visualized and imaged by Carl Zeiss Axio Imager D2 microscope using 63X Plan Apo 1.4 NA oil 


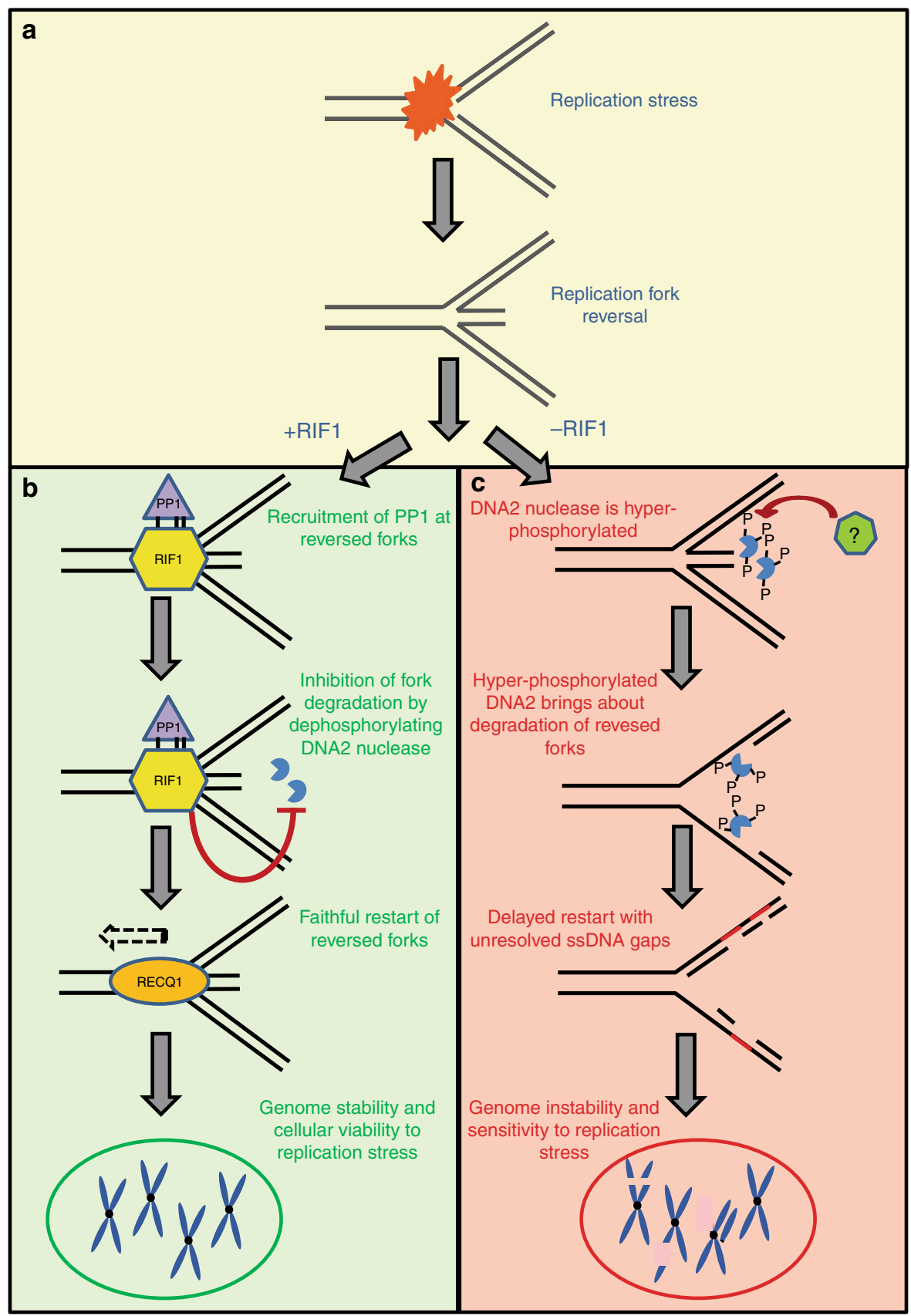

Fig. 7 Model for role of RIF1 in fork protection and genome stability. a Replication stress in cells results in replication fork reversal to stabilize stalled replication forks. b Fork reversal results in the recruitment of RIF1 probably through its C-terminal domain, which has cruciform structure binding properties. Binding of RIF1 to reversed forks stabilizes them by recruitment of PP1, which brings about de-phosphorylation of DNA2 and thereby limits access of DNA2 nuclease to these forks and prevents fork degradation. This allows for normal restart of reversed forks probably through RECQ1-mediated branch migration of these reversed forks resulting in prevention of genome instability and cellular viability upon replication stress. $\mathbf{c}$ In contrast, absence of RIF1 results in DNA2-mediated degradation of reversed forks. In the absence of the preferred substrate (four-way junctions), RECQ1 is unable to bind. Forks are therefore aberrantly restarted which results in exposure of under-replicated DNA in the form of ssDNA gaps behind the forks. These ssDNA gaps become a source of genome instability and DSBs later during the cell cycle in G2/M phases resulting in sensitivity to replication stress-inducing agents

immersion objective. Data analysis was carried out with ImageJ software64. The Mann-Whitney test was applied for statistical analysis using the GraphPad Prism Software.

Colony survival assay. Colony survival assay was performed according to the standard protocol as previously mentioned ${ }^{55}$. WT and Rif1 $1^{-l-}$ MEFs were seeded at low dilutions and treated with different replication poisons (HU, CPT, and Cisplatin) with different concentrations for $4 \mathrm{~h}$. In complementation experiments, first $\mathrm{Rif}^{-/-}$MEFs were transfected with hRIF deletion constructs along with full-length (hRIF1-FL, Del-HEAT, Del-CI, and Del-CII). The protein expression was allowed for $48 \mathrm{~h}$ and confirmed by western blotting. In parallel same cells were plated out at low dilutions and treated with drugs at different concentrations for $4 \mathrm{~h}$. Post treatment, drug treated medium was washed out and cells were allowed to grow in complete growth medium for 8 days. The colonies detected were fixed, stained, and subsequently analyzed with the Gel-counter by Oxford Optronix and appertaining Software (version 1.1.2.0). The survival was plotted after combining three independent experiments as the mean surviving percentage of colonies after drug treatment compared to the mean surviving colonies from the non-treated samples. 
Metaphase spreads and chromosomal aberrations. Metaphase spreads were carried out according to the standard protocol described previously ${ }^{8}$. Briefly, exponentially growing cells (50-80 \% confluence) were treated with drugs at different concentrations for $4 \mathrm{~h}$. Post treatment, drug treated medium was washed out and cells were allowed to grow in complete growth medium and exposed with colcemid for $6 \mathrm{~h}$. Metaphase spreads were prepared, stained by conventional methods. A minimum 60 metaphase images were using Carl Zeiss Axio Imager D2 microscope using 63x Plan Apo 1.4 NA oil immersion objective and analyzed with ImageJ software64 for chromosomal aberrations. Experiments were repeated three times. To determine the differences between conditions are significant a two-tailed $t$-test was used. $P$-values $<0.005\left(^{* * *}\right)$ and $<0.0005\left(^{* * *}\right)$ were considered as significant.

Immunofluorescence. Cells were grown on coverslips at $70-80 \%$ confluency. Cells were labeled with EdU $(10 \mu \mathrm{M})$ for $15 \mathrm{~min}$ to visualize cells in S-phase. After 15 min, cells were washed and incubated in fresh medium containing $\mathrm{HU}(4 \mathrm{mM})$ for $3 \mathrm{~h}$. After $3 \mathrm{~h}$ cells were washed in PBS and fixed in $2 \%$ paraformaldehyde in PBS containing $0.1 \%$ Triton-X and permeabilized for $15 \mathrm{~min}$ in $0.5 \%$ Triton-X in PBS. Coverslips were washed three times and subsequently stained with RIF1 primary antibody (1:5000, rabbit) for $1 \mathrm{~h}$ at RT. After $1 \mathrm{~h}$, cells were washed with PBS containing $0.1 \%$ Triton-X followed by incubation in secondary antibody conjugated to Alexafluor- 488 for $1 \mathrm{~h}$ at RT. EdU was visualized with a click-it reaction (click-it EdU imaging kit, Invitrogen) using a $594 \mathrm{nM}$ fluorescent azide according to the manufacturer's protocol. Coverslips were washed and incubated with DAPI (4',6-diamidino-2-phenylindole) for $10 \mathrm{~min}$ and mounted with ProLong Gold antifade reagent (Invitrogen). In experiments using ionizing radiations, cells were irradiated with ( $10 \mathrm{~Gy})$ and allowed to recover for $2 \mathrm{~h}$ followed by $2 \%$ paraformaldehyde fixation and immunostaining. Images were obtained using Carl Zeiss Axio Imager D2 microscope using 63X Plan Apo 1.4 NA oil immersion objective and analyzed with ImageJ software64. Details of the antibodies are provided in Supplementary Tables 1 and 2.

Electron microscope analysis. EM analysis was performed according to the standard protocol ${ }^{38}$. For DNA extraction, cells were lysed in lysis buffer and digested at $50^{\circ} \mathrm{C}$ in the presence of Proteinase- $\mathrm{K}$ for $2 \mathrm{~h}$. The DNA was purified using chloroform/isoamyl alcohol and precipitated in isopropanol and given 70\% ethanol wash and resuspended in elution buffer. Isolated genomic DNA was digested with PvuII HF restriction enzyme for 4 to $5 \mathrm{~h}$. Replication intermediates were enriched by using QIAGEN G-100 columns (as manufacture's protocol) and concentrated by an Amicon size-exclusion column. The benzyldimethylalkylammonium chloride (BAC) method was used to spread the DNA on the water surface and then loaded on carbon-coated nickel grids and finally DNA was coated with platinum using high-vacuum evaporator MED 010 (Bal Tec). Microscopy was performed with a transmission electron microscope FEI Talos, with $4 \mathrm{~K}$ by $4 \mathrm{~K}$ cmos camera. For each experimental condition, at least 70 replication fork intermediates were analyzed per experiment and ImageJ software64 was used to process analyze the images.

DSB detection by PFGE. DSB detection by PFGE was done as reported previously ${ }^{18}$. Briefly, cells were cast into $0.8 \%$ agarose plug $\left(2.5 \times 10^{5}\right.$ cells/plug $)$, digested in lysis buffer (100 mM EDTA, $1 \%$ sodium lauryl sarcosine, $0.2 \%$ sodium deoxycholate, $1 \mathrm{mg} / \mathrm{ml}$ proteinase- $\mathrm{K}$ ) at $37^{\circ} \mathrm{C}$ for $36-40 \mathrm{~h}$, and washed in $10 \mathrm{mM}$ Tris-HCl ( $\mathrm{pH} 8.0)-100 \mathrm{mM}$ EDTA. Electrophoresis was performed at $14^{\circ} \mathrm{C}$ in $0.9 \%$ pulse field-certified agarose (Bio-Rad) using Tris-borate-EDTA buffer in a Bio-Rad Chef DR III apparatus $\left(9 \mathrm{~h}, 120^{\circ}, 5.5 \mathrm{~V} / \mathrm{cm}\right.$, and 30- to 18 -s switch time; $6 \mathrm{~h}, 117^{\circ}, 4.5 \mathrm{~V} / \mathrm{cm}$, and 18 - to 9-s switch time; and $6 \mathrm{~h}, 112^{\circ}, 4 \mathrm{~V} / \mathrm{cm}$, and 9- to 5-s switch time). The gel was stained with ethidium bromide and imaged on UvidocHD2 Imager. Quantification of DSB was carried out using ImageJ software64. Relative DSB levels were calculated by comparing the results in the treatment conditions to that of the DSB level observed in untreated controls.

\section{Flow cytometry for cell cycle analysis and detection of RPA. For flow-} cytometric analysis of cell cycle, cells were labeled with EdU for 20 min followed by fixation for $10 \mathrm{~min}$ in $4 \%$ formaldehyde/PBS at room temperature. Cells were then washed with $1 \%$ BSA/PBS and permeabilized in $0.5 \%$ saponin buffer in $1 \%$ BSA/ PBS. Incorporated EdU was labeled according to the manufacturer's instructions (\#C35002; Invitrogen). Detection of RPA levels were carried out as previously described ${ }^{56}$. Briefly, cells were harvested after treatment with $4 \mathrm{mM} \mathrm{HU}$ in $1 \mathrm{~h}$ and the mentioned release time points. Pre-extraction was carried out in $0.2 \%$ Triton$\mathrm{X}-100 / \mathrm{PBS}$ to remove the non-chromatin bound RPA. Cells were then washed with $0.1 / \mathrm{BSA} / \mathrm{PBS}$ and fixed with $4 \%$ Paraformaldehyde. Permeabilization was carried out as discussed above, followed by staining with anti RPA32/2 antibody (\#ab2175, Abcam) for $1.5 \mathrm{~h}$ and subsequent incubation in secondary antibody. In both the assays, DNA was stained with $1 \mu \mathrm{g} / \mathrm{ml}$ DAPI. Samples were measured in BD LSR Fortessa and analyzed by FlowJo software v10.5.0. Details of the antibodies are provided in Supplementary Tables 1 and 2.

Proximity ligation-based assays (PLA). Cells were grown on cover slips until $60-70 \%$ confluency. Cells were incubated with $\mathrm{EdU}(20 \mu \mathrm{M})$ for $15 \mathrm{~min}$ to visualize cells in S-phase. After 15 min cells were washed and incubated in fresh media containing $\mathrm{HU}(4 \mathrm{mM})$ for $3 \mathrm{~h}$. After $3 \mathrm{~h}$ cells were washed two times with PBS and fixed with $2 \%$ paraformaldehyde for $15 \mathrm{~min}$ at room temperature. Following fixation and washing, cells were next permeabilized by $0.5 \%$ Triton-X in PBS for $15 \mathrm{~min}$ at room temperature. After two washes, freshly prepared click reaction mix ( $2 \mathrm{mM}$ copper sulfate, $10 \mu \mathrm{M}$ biotin-azide, and $100 \mathrm{mM}$ sodium ascorbate in PBS) was added to each samples and incubated in a humidified chamber at room temperature for $1 \mathrm{~h}$. After the click reaction, slides were washed with PBS for 5 min and placed back in humidified chamber with blocking buffer ( $10 \%$ goat serum and $0.1 \%$ Triton-X-100 in PBS) for $1 \mathrm{~h}$ at room temperature. Slides were incubated with primary antibody at $4{ }^{\circ} \mathrm{C}$ overnight in a humidified chamber. Mouse anti-biotin was used in conjunction with the RIF1 antibody. Slides were washed three times with wash buffer A (0.01 M Tris, $0.15 \mathrm{M} \mathrm{NaCl}$, and $0.05 \%$ Tween 20, pH 7.4) for 5 min each. Duolink In Situ PLA probes anti-mouse plus and anti-rabbit minus were diluted 1:5 in blocking solution ( $10 \%$ goat serum and $0.1 \%$ Triton-X-100 in PBS), dispensed onto slides $(30 \mu \mathrm{l} / \mathrm{well})$, and incubated for $1 \mathrm{~h}$ at $37^{\circ} \mathrm{C}$. Slides were again washed three times with buffer A 5 min each. Ligation mix was prepared by diluting Duolink ligation stock (1:5) and ligase (1:40) in high-purity water. Slides were placed back in the humid chamber, and ligation mix was dispensed onto slides $\left(30 \mu \mathrm{l} /\right.$ well) and incubated at $37^{\circ} \mathrm{C}$ for $30 \mathrm{~min}$ Slides were washed in $60 \mathrm{ml}$ wash buffer A two times for 2 min each. Amplification mix was prepared by diluting Duolink amplification stock (1:5) and rolling circle polymerase (1:80) in high-purity water. Slides were placed back in the humid chamber, and amplification mix was dispensed onto slides $(30 \mu \mathrm{l} /$ well $)$ and incubated at $37^{\circ} \mathrm{C}$ for $100 \mathrm{~min})$. Slides were washed with wash buffer B solution $(0.2 \mathrm{M}$ Tris and $0.1 \mathrm{M}$ $\mathrm{NaCl}$ ) three times for $10 \mathrm{~min}$ each and one time in $0.01 \times$ diluted wash buffer $\mathrm{B}$ solution for $1 \mathrm{~min}$, coverslips were incubated with DAPI for $5 \mathrm{~min}$ and mounted with ProLong Gold antifade reagent (Invitrogen). Slides were imaged using Zeiss LSM 700 Axio Imager Z2 confocal microscope and analyzed using Image software64.

Immunoprecipations. Cytoplasmic and nuclear extracts were prepared using NEPER Nuclear and Cytoplasmic extraction kit according to manufacturer's protocol. Protein concentrations were determined using BCA kit. Protein samples were precleared using $20 \mu \mathrm{l}$ of Protein A Sepharose Fast Flow (PAS) beads (GE Healthcare) by incubating $30 \mathrm{~min}$ at $4^{\circ} \mathrm{C}$. Samples were spun down and supernatant was collected in fresh tube. Ten percent inputs were taken out and $200 \mu \mathrm{g}$ of nuclear proteins were incubated with $2 \mu \mathrm{g}$ of DNA2 antibody along with respective IgG control over night $4{ }^{\circ} \mathrm{C}$ keeping final volume of $500 \mu \mathrm{l}$ with RIPA buffer $(0.01$ M Tris-HCl pH 7.5, $0.15 \mathrm{M} \mathrm{NaCl}, 1 \%$ Triton-X-100, $1 \% \mathrm{NP}-40,0.1 \%$ SDS, protease inhibitor cocktail). Next day, $50 \mu \mathrm{l}$ of Protein A Sepharose washed beads with 1XPBS $+0.1 \%$ NP40 was added and samples were further incubated for $2 \mathrm{~h}$ at $4{ }^{\circ} \mathrm{C}$ After final incubation samples were washed five times with wash buffer $(150 \mathrm{mM}$ $\mathrm{NaCl}, 20 \mathrm{mM}$ Tris-HCl (pH 7.5), 0.1\% NP-40, 1 mM EDTA, $2.5 \mathrm{mM}$ Sodium pyrophosphate, protease inhibitor) $500 \mu \mathrm{l}$ each, and eluted in $2 \mathrm{x}$ Laemmli sample buffer for sodium dodecyl sulfate polyacrylamide gel electrophoresis and immunoblot.

Plasmid-based DNA repair assays. For determining the HR and NHEJ proficiency in WT and Rif1-l- cells the following plasmids were used: pDRGFP (Addgene plasmid \#26475) and pCBASceI(Addgene plasmid \#26477) for HR and pCVL Traffic Light Reporter1.1(Sce target) (Addgene plasmid \#31482) along with pCBASceI for NHEJ. For measuring and controlling transfection efficiency, TurboGFP expressing plasmid (Sigma, MISSION SHC003) and Scrambled plasmid (Sigma, MISSION SHC002), were used. In all, $2.5 \times 10^{5}$ cells were co-transfected with $3 \mu \mathrm{g}$ of plasmid combinations (i.e., $1.5 \mu \mathrm{g}$ of each plasmid) using Xtremegene9 reagent from Roche in six-well dish. Cells were transfected twice at an interval of $24 \mathrm{~h}$. Post $48 \mathrm{~h}$ of transfection, cells were harvested and the GFP-positive cells (for HR) and RFP-positive cells (for NHEJ) were assessed by flow cytometry. Fifty thousand events were recorded for each sample. Background normalization was done by using samples co-transfected with scrambled-reporter plasmid and also scrambled-pCBASceI plasmid. Final percentage of GFP and RFP-positive cells were calculated based on the transfection efficiency of the cells. Each experiment was independently performed at least thrice.

Reporting summary. Further information on research design is available in the Nature Research Reporting Summary linked to this article.

\section{Data availability}

The iPOND dataset has been deposited in Figshare repository [https://doi.org/10.6084/ m9.figshare.8242088.v1]. The source data underlying Figs. 3a, 4b, g, 5a, 6a, and Supplementary Figs. 1b, 2a, b, e, 3c, f, g, 4c, and 6a, c are provided as a Source Data file. A reporting summary for this Article is included as Supplementary Information file.

Received: 30 July 2018 Accepted: 1 July 2019

Published online: 23 July 2019 


\section{References}

1. Moldovan, G. L. \& D'Andrea, A. D. How the fanconi anemia pathway guards the genome. Annu. Rev. Genet. 43, 223-249 (2009).

2. Venkitaraman, A. R. Cancer susceptibility and the functions of BRCA1 and BRCA2. Cell 108, 171-182 (2002).

3. Schlacher, K. et al. Double-strand break repair-independent role for BRCA2 in blocking stalled replication fork degradation by MRE11. Cell 145, 529-542 (2011).

4. Schlacher, K., Wu, H. \& Jasin, M. A distinct replication fork protection pathway connects Fanconi anemia tumor suppressors to RAD51-BRCA1/2. Cancer Cell 22, 106-116 (2012).

5. Ding, $X$. et al. Synthetic viability by BRCA2 and PARP1/ARTD1 deficiencies. Nat. Commun. 7, 12425 (2016).

6. Ray Chaudhuri, A. et al. Replication fork stability confers chemoresistance in BRCA-deficient cells. Nature 535, 382-387 (2016).

7. Higgs, M. R. et al. BOD1L is required to suppress deleterious resection of stressed replication forks. Mol. Cell 59, 462-477 (2015).

8. $\mathrm{Xu}, \mathrm{S}$. et al. Abro1 maintains genome stability and limits replication stress by protecting replication fork stability. Genes Dev. 31, 1469-1482 (2017).

9. Kolinjivadi, A. M. et al. Smarcal1-mediated fork reversal triggers Mre11dependent degradation of nascent DNA in the absence of Brca2 and stable Rad51 nucleofilaments. Mol. Cell 67, 867-881 e867 (2017).

10. Lemacon, D. et al. MRE11 and EXO1 nucleases degrade reversed forks and elicit MUS81-dependent fork rescue in BRCA2-deficient cells. Nat. Commun. 8, 860 (2017)

11. Mijic, S. et al. Replication fork reversal triggers fork degradation in BRCA2defective cells. Nat. Commun. 8, 859 (2017).

12. Taglialatela, A. et al. Restoration of replication fork stability in BRCA1- and BRCA2-deficient cells by inactivation of SNF2-family fork remodelers. Mol. Cell 68, 414-430 e418 (2017)

13. Follonier, C., Oehler, J., Herrador, R. \& Lopes, M. Friedreich's ataxiaassociated GAA repeats induce replication-fork reversal and unusual molecular junctions. Nat. Struct. Mol. Biol. 20, 486-494 (2013)

14. Neelsen, K. J. \& Lopes, M. Replication fork reversal in eukaryotes: from dead end to dynamic response. Nat. Rev. Mol. Cell Biol. 16, 207-220 (2015).

15. Neelsen, K. J., Zanini, I. M., Herrador, R. \& Lopes, M. Oncogenes induce genotoxic stress by mitotic processing of unusual replication intermediates. $J$. Cell Biol. 200, 699-708 (2013).

16. Ray Chaudhuri, A. et al. Topoisomerase I poisoning results in PARP-mediated replication fork reversal. Nat. Struct. Mol. Biol. 19, 417-423 (2012)

17. Chapman, J. R. et al. RIF1 is essential for 53BP1-dependent nonhomologous end joining and suppression of DNA double-strand break resection. Mol. Cell 49, 858-871 (2013).

18. Cornacchia, D. et al. Mouse Rif1 is a key regulator of the replication-timing programme in mammalian cells. EMBO J. 31, 3678-3690 (2012).

19. Di Virgilio, M. et al. Rif1 prevents resection of DNA breaks and promotes immunoglobulin class switching. Science 339, 711-715 (2013).

20. Escribano-Diaz, C. et al. A cell cycle-dependent regulatory circuit composed of 53BP1-RIF1 and BRCA1-CtIP controls DNA repair pathway choice. Mol. Cell 49, 872-883 (2013)

21. Feng, L., Fong, K. W., Wang, J., Wang, W. \& Chen, J. RIF1 counteracts BRCA1-mediated end resection during DNA repair. J. Biol. Chem. 288, 11135-11143 (2013).

22. Foti, R. et al. Nuclear architecture organized by Rif1 underpins the replicationtiming program. Mol. Cell 61, 260-273 (2016).

23. Hengeveld, R. C. et al. Rif1 is required for resolution of ultrafine DNA bridges in anaphase to ensure genomic stability. Dev. Cell 34, 466-474 (2015).

24. Hiraga, S. I. et al. Human RIF1 and protein phosphatase 1 stimulate DNA replication origin licensing but suppress origin activation. EMBO Rep. 18, 403-419 (2017).

25. Zimmermann, M., Lottersberger, F., Buonomo, S. B., Sfeir, A. \& de Lange, T. 53BP1 regulates DSB repair using Rif1 to control 5' end resection. Science 339, 700-704 (2013).

26. Callen, E. et al. 53BP1 mediates productive and mutagenic DNA repair through distinct phosphoprotein interactions. Cell 153, 1266-1280 (2013).

27. Buonomo, S. B., Wu, Y., Ferguson, D. \& de Lange, T. Mammalian Rif1 contributes to replication stress survival and homology-directed repair. J. Cell Biol. 187, 385-398 (2009).

28. Ahuja, A. K. et al. A short G1 phase imposes constitutive replication stress and fork remodelling in mouse embryonic stem cells. Nat. Commun. 7, 10660 (2016).

29. Dungrawala, H. et al. RADX promotes genome stability and modulates chemosensitivity by regulating RAD51 at replication forks. Mol. Cell 67, 374-386 e375 (2017)

30. Dungrawala, $H$. et al. The replication checkpoint prevents two types of fork collapse without regulating replisome stability. Mol. Cell 59, 998-1010 (2015).
31. Her, J., Ray, C., Altshuler, J., Zheng, H. \& Bunting, S. F. 53BP1 mediates ATRChk1 signaling and protects replication forks under conditions of replication stress. Mol. Cell. Biol. 38, https://doi.org/10.1128/MCB.00472-17 (2018).

32. Bunting, S. F. et al. 53BP1 inhibits homologous recombination in Brca1deficient cells by blocking resection of DNA breaks. Cell 141, 243-254 (2010).

33. Pierce, A. J., Johnson, R. D., Thompson, L. H. \& Jasin, M. XRCC3 promotes homology-directed repair of DNA damage in mammalian cells. Genes Dev. 13, 2633-2638 (1999)

34. Findlay, S. et al. SHLD2/FAM35A co-operates with REV7 to coordinate DNA double-strand break repair pathway choice. EMBO J. 37, https://doi.org/ 10.15252/embj.2018100158 (2018).

35. Isobe, S. Y., Nagao, K., Nozaki, N., Kimura, H. \& Obuse, C. Inhibition of RIF1 by SCAI allows BRCA1-mediated repair. Cell Rep. 20, 297-307 (2017)

36. Zellweger, R. \& Lopes, M. Dynamic architecture of eukaryotic DNA replication forks in vivo, visualized by electron microscopy. Methods Mol. Biol. 1672, 261-294 (2018)

37. Thangavel, S. et al. DNA2 drives processing and restart of reversed replication forks in human cells. J. Cell Biol. 208, 545-562 (2015).

38. Zellweger, R. et al. Rad51-mediated replication fork reversal is a global response to genotoxic treatments in human cells. J. Cell Biol. 208, 563-579 (2015).

39. Bhat, K. P. et al. RADX modulates RAD51 activity to control replication fork protection. Cell Rep. 24, 538-545 (2018).

40. Sukackaite, R. et al. Mouse Rif1 is a regulatory subunit of protein phosphatase 1 (PP1). Sci. Rep. 7, 2119 (2017).

41. Shibata, A. et al. DNA double-strand break repair pathway choice is directed by distinct MRE11 nuclease activities. Mol. Cell 53, 7-18 (2014).

42. Kumar, S. et al. Inhibition of DNA2 nuclease as a therapeutic strategy targeting replication stress in cancer cells. Oncogenesis 6, e319 (2017).

43. Sukackaite, R. et al. Structural and biophysical characterization of murine rif C terminus reveals high specificity for DNA cruciform structures. J. Biol. Chem. 289, 13903-13911 (2014).

44. Alver, R. C., Chadha, G. S., Gillespie, P. J. \& Blow, J. J. Reversal of DDKmediated MCM phosphorylation by Rif1-PP1 regulates replication initiation and replisome stability independently of ATR/Chk1. Cell Rep. 18, 2508-2520 (2017).

45. Choy, M. S. et al. PP1:tautomycetin complex reveals a path toward the development of PP1-specific inhibitors. J. Am. Chem. Soc. 139, 17703-17706 (2017).

46. Lopes, M., Foiani, M. \& Sogo, J. M. Multiple mechanisms control chromosome integrity after replication fork uncoupling and restart at irreparable UV lesions. Mol. Cell 21, 15-27 (2006).

47. Ge, X. Q. \& Blow, J. J. Chk1 inhibits replication factory activation but allows dormant origin firing in existing factories. J. Cell Biol. 191, 1285-1297 (2010).

48. $\mathrm{Xu}, \mathrm{D}$. et al. Rif1 provides a new DNA-binding interface for the Bloom syndrome complex to maintain normal replication. EMBO J. 29, 3140-3155 (2010).

49. Xu, Y. et al. 53BP1 and BRCA1 control pathway choice for stalled replication restart. Elife 6, https://doi.org/10.7554/eLife.30523 (2017).

50. Vriend, L. E., Jasin, M. \& Krawczyk, P. M. Assaying break and nick-induced homologous recombination in mammalian cells using the DR-GFP reporter and Cas9 nucleases. Methods Enzym. 546, 175-191 (2014).

51. Rondinelli, B. et al. EZH2 promotes degradation of stalled replication forks by recruiting MUS81 through histone H3 trimethylation. Nat. Cell Biol. 19, 1371-1378 (2017)

52. Berti, M. et al. Human RECQ1 promotes restart of replication forks reversed by DNA topoisomerase I inhibition. Nat. Struct. Mol. Biol. 20, 347-354 (2013).

53. Hashimoto, Y., Chaudhuri, A. R., Lopes, M. \& Costanzo, V. Rad51 protects nascent DNA from Mre11-dependent degradation and promotes continuous DNA synthesis. Nat. Struct. Mol. Biol. 17, 1305-1311 (2010).

54. Cox, J. et al. A practical guide to the MaxQuant computational platform for SILAC-based quantitative proteomics. Nat. Protoc. 4, 698-705 (2009).

55. Tripathi, V., Nagarjuna, T. \& Sengupta, S. BLM helicase-dependent and-independent roles of 53BP1 during replication stress-mediated homologous recombination. J. Cell Biol. 178, 9-14 (2007).

56. Forment, J. V., Walker, R. V. \& Jackson, S. P. A high-throughput, flow cytometry-based method to quantify DNA-end resection in mammalian cells. Cytom. A 81, 922-928 (2012).

\section{Acknowledgements}

We thank Roland Kanaar, Andre Nussenzweig, Massimo Lopes, and Nitika Taneja for stimulating discussions and sharing important reagents used in the manuscript; Alessandro Vindigni and Grzegorz Ira for DNA2i, Michela Di Virgilio for mouse RIF1 antibody, Calvin Lo for help with flow cytometry, Przemek Krawczyk for constructs, Ross Chapman and Simon Boulton for cell lines. This work was supported by grants from the Netherlands Organization for Scientific research (NWO-VIDI \#91713334) and the 
European Research Council (ERC CoS Grant 682421) to MATMvV, a Dutch Cancer Society (KWF grant 11008/2017-1) grant, Erasmus MC Daniel den Hoed instrument grant and startup funds from the Erasmus MC to ARC.

\section{Author contributions}

C.M. conducted all the fiber experiments, PFGE, FACS, and cloning experiments. V.T. performed clonogenic assays, metaphase spreads, PLA and immunofluorescence experiments. E.M.M. performed the EM experiments with help from C.M. A.M.H. and H.R.d.B. performed SCE experiments. G.R. performed immunofluorescence experiments for RAD51. S.M. helped C.M. in cloning experiments. J.D. analyzed mass-spectrometry data. M.A.T.M.v.V supervised the SCE experiments and helped in finalizing the manuscript. A.R.C. conceptualized the project, supervised it, and wrote the manuscript.

\section{Additional information}

Supplementary Information accompanies this paper at https://doi.org/10.1038/s41467019-11246-1.

Competing interests: The authors declare no competing interests.

Reprints and permission information is available online at http://npg.nature.com/ reprintsandpermissions/
Peer review information: Nature Communications thanks the anonymous reviewer(s) for their contribution to the peer review of this work. Peer reviewer reports are available.

Publisher's note: Springer Nature remains neutral with regard to jurisdictional claims in published maps and institutional affiliations.

(c) (i) Open Access This article is licensed under a Creative Commons Attribution 4.0 International License, which permits use, sharing, adaptation, distribution and reproduction in any medium or format, as long as you give appropriate credit to the original author(s) and the source, provide a link to the Creative Commons license, and indicate if changes were made. The images or other third party material in this article are included in the article's Creative Commons license, unless indicated otherwise in a credit line to the material. If material is not included in the article's Creative Commons license and your intended use is not permitted by statutory regulation or exceeds the permitted use, you will need to obtain permission directly from the copyright holder. To view a copy of this license, visit http://creativecommons.org/ licenses/by/4.0/.

(c) The Author(s) 2019 